\title{
Samelson products and exponents of homotopy groups
}

\author{
Joseph A. Neisendorfer
}

Received: 27 June 2012 / Accepted: 5 December 2012 / Published online: 15 December 2012 (C) Tbilisi Centre for Mathematical Sciences 2012

\begin{abstract}
This paper is an introduction to homotopy groups with coefficients, their Samelson products, and their main applications to higher order torsion in the homotopy groups of Moore spaces and to exponents of the homotopy groups of spheres. All this material is treated in the author's book (Neisendorfer in Algebraic methods in unstable homotopy theory, Cambridge University Press, 2009). Other useful references are the author's memoir, Primary homotopy theory (Neisendorfer in Mem AMS 232, American Mathematical Society, 1980), and the author's paper, Homotopy groups with coefficients (Neisendorfer in J Fixed Point Theory Appl 8:247-338, 2010).
\end{abstract}

Keywords Samelson products $\cdot$ Homotopy groups $\cdot$ Exponents

Mathematics Subject Classification (2000) Primary 55-02 · 55Q40 - 55Q15;

Secondary 55R05

\section{Contents}

Introduction . . . . . . . . . . . . . . . . . . . . . . . . 240

2 Homotopy groups with coefficients . . . . . . . . . . . . . . . . . . . . . . . . . 242

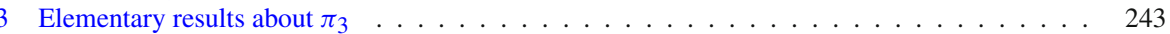

4 Bouquet splittings of smash products of Moore spaces . . . . . . . . . . . . . . . . . . . . . 244

5 Reduction and inflation maps . . . . . . . . . . . . . . . . . . . . . . . . . . . . . . 245

6 Universal coefficients and homotopy Bockstein spectral sequences . . . . . . . . . . . . . . . 246

7 Graded Lie algebras . . . . . . . . . . . . . . . . . . . . . . . . . . . . . . . . . . . . . 247

Communicated by Jim Stasheff.

J. A. Neisendorfer $(\bowtie)$

Department of Mathematics, University of Rochester, Rochester, NY 14625, USA

e-mail: jnei@math.rochester.edu 
8 Samelson products and differential Lie identities _. . . . . . . . . . . . . . . . . . 250

9 Compressions of Samelson products . . . . . . . . . . . . . . . . . . . . . . . 251

$10 \mathrm{H}$-based compressions of Samelson products . . . . . . . . . . . . . . . . . . . . . . . . 254

11 Universal models for relative Samelson products . . . . . . . . . . . . . . . . . . . . . 256

12 Universal models for H-based Samelson products . . . . . . . . . . . . . . . . . . . . . . . 258

13 Lie identities for H-based Samelson products . . . . . . . . . . . . . . . . . . . . . . . 259

14 Higher order torsion in the homotopy groups of a Moore space . . . . . . . . . . . . . . . . 262

15 Twisted tensor products and the fibre of the pinch map . . . . . . . . . . . . . . . . . 265

16 Lie algebras and the loops on the fibre of the pinch map . . . . . . . . . . . . . . . . . . . 267

17 The $\bmod p$ homology Bockstein spectral sequence of the loops

on the fibre of the pinch map . . . . . . . . . . . . . . . . . . . . . . 268

18 A tensor product splitting of a universal enveloping algebra . . . . . . . . . . . . . . . . . 270

19 Product splitting of the loops on the fibre of the pinch map . . . . . . . . . . . . . . . . 273

20 Exponents of the homotopy groups of spheres . . . . . . . . . . . . . . . . . . . 275

\section{Introduction}

Homotopy groups with coefficients were introduced by Frank Peterson in his thesis. This survey presents the basic properties of these groups and their more advanced theory of Samelson products, including the theories of relative Samelson products and $\mathrm{H}$-based Samelson products in fibration sequences. These latter theories were new even in the classical case of integral homotopy groups.

It is elementary that relative and $\mathrm{H}$-based Samelson products both factor through the fibre in the loops on fibrations sequences. What requires care is that the Lie identities of anti-commutativity and the Jacobi identity are valid.

Here is a personal note. I have never had much doubt that the Lie identities were valid for relative Samelson products. But I have feared that the Lie identities could fail for H-based Samelson products. These latter products depend on the base of the fibration sequence being the loops on an $\mathrm{H}$-space. When I wrote my book, I wrote the section on H-based products with a neurotic obsession on verification of the Lie identities. Over time, Brayton Gray has provided a valuable interrogation of the validity of this work. It has survived this rigorous test. But in the process, I have learned that, in my search for certainty, I did not present the theory of H-based Samelson products in the clearest possible way. I hope this paper remedies that.

My worries have been much diminished by a revelation that I had while walking on a Lake Erie beach. I realized that Quillen's Lie algebra model category for rational homotopy theory yields undeniable proofs of the Lie identities for both relative and $\mathrm{H}$ based Samelson products in rational homotopy theory. Of course, rational homotopy theory is not sufficient for the main applications but it is a comfort.

In my opinion, it has not been stressed enough that the nonexistence of elements of Hopf invariant one and of mod p Hopf invariant one are the obstacles to Lie identities for Samelson products in classical integral homotopy groups unless the primes 2 and 3 are inverted. In order to embed a graded Lie algebra in a universal enveloping algebra, there must be a squaring operation for odd dimensional classes. The existence of this squaring operation is automatic if the prime 2 is inverted. But, if 2 is not inverted, Hopf invariant one forbids the general existence of this squaring operation.

Samelson products in homotopy groups with coefficients have profound difficulties when the coefficients are cyclic groups of orders a power of 2 or 3 . With mod 2 
coefficients these products do not even exist. With 2 primary coefficients mod 4 or greater they exist but the Lie identities may not be valid. It would be a good thing to clarify the 2 primary situation. With 3 primary coefficients the problem is that the Jacobi identity and a certain triple vanishing condition both fail. The first fact was pointed out to me by Toda when I was writing my thesis.

Accordingly, we have for the most part ignored the primes 2 and 3 in this paper. With hard technical work, the problems of the exponents of the homotopy groups of Moore spaces and of spheres have both been solved at the prime 3. Although Selick has improved Toda's upper bound for the 2 primary components of the homotopy group of odd dimensional spheres, there is no proof of the Barratt-Mahowald conjecture and the best possible exponent is still unsolved in this case. To my knowledge, no one is working on it anymore.

We close by giving the two principle applications of Samelson products in fibration sequences. First, the relative products are used to show the existence of higher order torsion in the integral homotopy groups of odd primary Moore spaces. An odd primary mod $p^{r}$ Moore space has infinitely many elements of order $p^{r+1}$ in its integral homotopy groups. Second, the H-based products are used to prove the exponent theorem for the odd primary components of the homotopy groups of odd dimensional spheres. A $2 n+1$ dimensional sphere has all odd primary torsion in its homotopy groups annihilated by $p^{n}$. For simplicity, we restrict the presentation here to primes greater than 3 .

Properties of graded Lie algebras are central to these two applications. For example, subalgebras of free algebras are free. Techniques are given for determining generators for these subalgebras. We have described these results but we have not proved them here. The same is true for the Eilenberg-Moore results on the homology of fibrations of both spaces and of differential coalgebras. Perhaps it is true that we achieve a greater clarity and flow by omitting these proofs. Certainly, we tire the reader less. Nonetheless, these algebraic techniques are worth study.

The homotopy theoretic fibre of the pinch map from a mod $p^{r}$ Moore space to a sphere plays a central role in both the higher order homotopy torsion result for odd primary Moore spaces and in the odd primary exponent theorem for the homotopy groups of spheres. First, we show the surprising result that the homology of this fibre is torsion free. Then we show via a twisted tensor product that the homology of the fibre is weakly equivalent as a differential coalgebra to the chains on the fibre.

This result is used to show that the homology of the loops on the fibre can be computed via the cobar construction on the homology of the fibre. If we compare this cobar construction with the cobar construction which computes the homology of the double loops on a sphere, we get that the homology of the loops on the fibre has $p$ torsion of order less than or equal to $p^{r+1}$. The $r$-th Bockstein differential allows the mod p homology of the loops on a Moore space to be be regarded as an acyclic twisted tensor product. Combined with the previous upper bound on the order of the torsion, this gives the computation of the mod $p$ homology Bockstein spectral sequence of the loops on the fibre.

The mod $p$ homology Bockstein spectral sequence of the loop space of the fibre determines the order of the torsion in the homotopy of odd primary Moore spaces. This spectral sequence leads to an algebraic tensor product decomposition of the homology 
of this loop space which has a geometric realization by a product decomposition of the localized loop space. This product decomposition yields the odd primary exponent theorem for spheres.

\section{Homotopy groups with coefficients}

Definition 2.1 The Moore spaces (or as he called them, Peterson spaces), with one nontrivial cohomology group isomorphic to $Z / k Z$ in dimension $n$, are the domains of the $\bmod k$ homotopy groups,

$$
P^{n}(k)=S^{n-1} \cup_{k} e^{n},
$$

These are suspensions if $n \geq 3$ and double suspensions if $n \geq 4$. That is,

$$
\pi_{n}(X ; Z / k Z)=\left[P^{n}(k), X\right]_{*}=\text { group if } n \geq 3 \text {, abelian group if } n \geq 4
$$

and classically

$$
\pi_{n}(X ; Z)=\pi_{n}(X)=\left[S^{n}, X\right]_{*}=\text { group if } n \geq 1 \text {, abelian group if } n \geq 2 \text {. }
$$

In the next section we show

Theorem $2.2 \pi_{n}(X ; Z / k Z), n \geq 3$ is an abelian group if $k$ is odd and it is annihilated by $k$ if $k$ is odd or divisible by 4 .

Remark 2.3 But $\pi_{3}\left(X, Z / 2^{r} Z\right)$ need not be abelian and $\pi_{3}(X ; Z / 2 Z)$ may have elements of order 4 .

Recall

$$
\begin{array}{r}
H_{n-1}\left(P^{n}(k) ; Z\right)=Z / k Z, \quad H^{n}\left(P^{n}(k) ; Z\right)=Z / k Z, \\
H_{\ell}\left(P^{n}(k) ; Z / k Z\right)=Z / k Z, \quad H^{\ell}\left(P^{n}(k) ; Z / k Z\right)=Z / k Z, \ell=n-1, n .
\end{array}
$$

We will often indicate generators of these groups by using the notation $e_{n}$ where the $n$ represents the dimension of this generator.

Definition 2.4 The mod $k$ Hurewicz map

$$
\phi: \pi_{n}(X ; Z / k Z) \rightarrow H_{n}(X ; Z / k Z), \quad n \geq 2
$$

is the natural transformation defined by $\phi[f]=f_{*} e_{n}$ where $e_{n}$ is a canonical generator of $H_{n}\left(P^{n}(k) ; Z / k Z\right)=Z / k Z$.

We have a Hurewicz theorem [16,19].

Theorem 2.5 If X is simplyconnected, $n \geq 2$, and $\pi_{k}(X ; Z / k Z)=0$ for all $k \leq n-1$, then $\phi: \pi_{n}(X ; Z / k Z) \rightarrow H_{n}(X ; Z / k Z)$ is a bijection. 


\section{Elementary results about $\pi_{3}$}

The abelian group and exponent results below are true for all $\pi_{n}, n \geq 3$ but dimension 3 is the crucial case. The abelian condition is automatic in the higher dimensions and the exponent result follows by suspension of the domain.

Lemma 3.1 If $k$ is an odd prime, then $\pi_{3}(X ; Z / k Z)$ is an abelian group and it is annihilated by $k$ if $k$ is odd or divisible by 4 .

Proof We prove this by using the following result which can be found in Steenrod and Epstein [25].

Lemma 3.2 The reduced diagonal $\Delta: P^{2}(k) \rightarrow P^{2}(k) \wedge P^{2}(k)$ induces $\Delta_{*}\left(e_{2}\right)=$ $\frac{k(k-1)}{2} e_{1} \otimes e_{1}$ in $\bmod k$ homology.

Thus, the mod $k$ Hurewicz theorem implies that it is null homotopic if $k$ is odd. Hence, the composition

$$
P^{2}(k) \stackrel{\Delta}{\rightarrow} P^{2}(k) \wedge P^{2}(k) \stackrel{f \wedge g}{\longrightarrow} G \wedge G \stackrel{[\quad, \quad]}{\longrightarrow} G
$$

is null homotopic for all grouplike spaces $G$ where $[x, y]=x^{-1} y^{-1} x y$ is the commutator map.

If $G=\Omega X$, then $\pi_{2}(G ; Z / k Z)=\pi_{3}(X ; Z / k Z)$ is abelian whenever $k$ is odd.

Recall the classical Hopf invariant $[8,25]$ of a map $\alpha: S^{3} \rightarrow S^{2}$ :

$$
e_{2} \cup e_{2}=H(\alpha) e_{4}
$$

in the integral cohomology of the mapping cone

$$
S^{2} \cup_{\alpha} e^{4}
$$

Theorem 3.3 (Hopf) $H: \pi_{3}\left(S^{2}\right) \rightarrow Z$ is an isomorphism.

Suppose $\alpha: P^{3}(k) \rightarrow P^{3}(k)$ is a map which induces 0 in $\bmod k$ homology. There is a $\bmod k$ Hopf invariant [19]

$$
e_{2} \cup e_{2}=H(\alpha) e_{4}
$$

in the $\bmod k$ cohomology of the mapping cone

$$
P^{3}(k) \cup_{\alpha} C P^{3}(k)
$$

Theorem $3.4 H: \overline{\pi_{3}}\left(P^{3}(k)\right) \rightarrow Z / k Z$ is an isomorphism where $\overline{\pi_{3}}\left(P^{3}(k) ; Z / k Z\right)$ $i$ the subgroup of all homotopy classes which induce 0 on mod $k$ homology. 
Consider the map $k: S^{1} \rightarrow S^{1}$ and the cofibration sequence

$$
S^{1} \wedge P^{2}(k) \stackrel{k=k \wedge 1}{\longrightarrow} S^{1} \wedge P^{2}(k) \rightarrow P^{2}(k) \wedge P^{2}(k)
$$

Since $\left(e_{1} \otimes e_{1}\right)^{2}=-\left(\frac{k(k-1)}{2}\right)^{2}\left(e_{2} \otimes e_{2}\right)$, the $\bmod k$ Hopf invariant is

$$
H(k)=-\left(\frac{k(k-1)}{2}\right)^{2}
$$

which is 0 in $Z / k Z$ if $k$ is odd or divisible by 4 .

Hence, $k$ is 0 in $\pi_{3}\left(P^{3}(k) ; Z / k Z\right)$ and by naturality multiplication by $k$ is 0 in all $\pi_{3}(X ; Z / k Z)$.

Remark 3.5 By suspension $k$ is zero in all $\pi_{n}\left(P^{n}(k) ; Z / k Z\right)$ for $n \geq 3$. It follows from naturality that multiplication by $k$ is 0 in all $\pi_{n}(X ; Z / k Z)$ if $n \geq 3$.

\section{Bouquet splittings of smash products of Moore spaces}

The exponent theorems in the previous section lead immediately to the decompositions of smash products of Moore spaces. These decompositions are essential for defining a Samelson product in homotopy groups with coefficients.

Theorem 4.1 If $k$ is odd or divisible by 4 and $n, m \geq 2$, then there is a homotopy equivalence

$$
P^{n+m}(k) \vee P^{n+m-1}(k) \stackrel{\Delta \vee \iota}{\longrightarrow} P^{n}(k) \wedge P^{m}(k) .
$$

Proof In the cofibration sequence

$$
\begin{aligned}
S^{n-1} & \wedge P^{m}(k) \stackrel{k=k \wedge 1}{\rightarrow} S^{n-1} \wedge P^{m}(k) \stackrel{\iota}{\rightarrow} P^{n}(k) \\
& \wedge P^{m}(k) \stackrel{j}{\rightarrow} S^{n} \wedge P^{m}(k) \stackrel{k}{\rightarrow} S^{n} \wedge P^{m}(k) \rightarrow \cdots
\end{aligned}
$$

the two maps $k$ are null homotopic. (Here, $\iota$ is the map which appears in the statement of the theorem.) Hence there is a map $\Delta: S^{n} \wedge P^{m}(k)=P^{n+m}(k) \rightarrow P^{n}(k) \wedge P^{m}(k)$ which is a section, that is, $j \cdot \Delta=1$. Since $\iota \vee \Delta: P^{n+m-1}(k) \vee P^{n+m}(k) \rightarrow$ $P^{n}(k) \wedge P^{m}(k)$ is a homology equivalence of simply connected spaces, it is a homotopy equivalence.

Let $p$ be an odd prime and let

$$
T: P^{n}\left(p^{r}\right) \wedge P^{m}\left(p^{r}\right) \rightarrow P^{m}\left(p^{r}\right) \wedge P^{n}\left(p^{r}\right), \quad T(x \wedge y)=y \wedge x
$$


be the twist map. Consider the diagrams

$$
\begin{aligned}
& P^{n+m}\left(p^{r}\right) \quad \stackrel{\Delta}{\rightarrow} \quad P^{n}\left(p^{r}\right) \wedge P^{m}\left(p^{r}\right) \\
& \downarrow(-1)^{n m} \quad \downarrow T \\
& P^{n+m}\left(p^{r}\right) \quad \stackrel{\Delta}{\rightarrow} \quad P^{m}\left(p^{r}\right) \wedge P^{n}\left(p^{r}\right) \\
& P^{n+m+q}\left(p^{r}\right) \quad \stackrel{\Delta}{\rightarrow} \quad P^{n}\left(p^{r}\right) \wedge P^{m+q}\left(p^{r}\right) \\
& \downarrow \Delta \quad \downarrow 1 \wedge \Delta \\
& P^{n+m}\left(p^{r}\right) \wedge P^{q}\left(p^{r}\right) \stackrel{\Delta \wedge 1}{\longrightarrow} P^{n}\left(p^{r}\right) \wedge P^{m}\left(p^{r}\right) \wedge P^{q}\left(p^{r}\right)
\end{aligned}
$$

We assert without proof [19] that the maps $\Delta: P^{n+m}\left(p^{r}\right) \rightarrow P^{n}\left(p^{r}\right) \wedge P^{m}\left(p^{r}\right)$ are cocommutative and, if $p$ is greater than 3 , coassociative in the sense that the above diagrams are homotopy commutative, except for the addition of some compositions with Whitehead products for small values of $n, m, q$.

If $p$ is greater than 3 , it follows that these diagrams become homotopy commutative in all cases if they are augmented by a map of the lower right hand corners into an $\mathrm{H}$ space.

\section{Reduction and inflation maps}

We shall see that so-called reduction maps and inflation maps play a role in subsequent applications to exponent theorems for Moore spaces and spheres. These maps are the duals of the following.

The coinflation maps $\bar{\eta}$ and the coreduction maps $\bar{\rho}$ are defined by the maps of horizontal cofibration sequences below

$$
\begin{aligned}
& S^{n-1} \stackrel{k \ell}{\rightarrow} S^{n-1} \stackrel{\iota}{\rightarrow} P^{n}(k \ell) \stackrel{q}{\rightarrow} S^{n} \\
& \downarrow \ell \quad \downarrow 1 \quad \downarrow \bar{\eta} \quad \downarrow \ell \\
& S^{n-1} \stackrel{k}{\rightarrow} S^{n-1} \stackrel{\iota}{\rightarrow} P^{n}(k) \stackrel{q}{\rightarrow} S^{n} \\
& S^{n-1} \stackrel{k}{\rightarrow} S^{n-1} \stackrel{\iota}{\rightarrow} P^{n}(k) \stackrel{q}{\rightarrow} S^{n} \\
& \downarrow 1 \quad \downarrow \ell \quad \downarrow \bar{\rho} \quad \downarrow 1 \\
& S^{n-1} \stackrel{k \ell}{\rightarrow} S^{n-1} \stackrel{\iota}{\rightarrow} P^{n}(k \ell) \stackrel{q}{\rightarrow} S^{n}
\end{aligned}
$$

Thus, the coinflation maps are degree 1 on the bottom cell and degree $\ell$ on the top cell. The coreduction maps are degree $\ell$ on the bottom cell and degree 1 on the top cell.

Definition 5.1 The dual of the coreduction map is the reduction map $\rho: \pi_{*}(X ; Z /$ $k \ell Z) \rightarrow \pi_{*}(X ; Z / k Z)$ and the Bocksteins $\beta: \pi_{*}(X ; Z / k Z) \rightarrow \pi_{*-1}(X)$ are related by $\ell \cdot \beta=\beta \cdot \rho: \pi_{*}(X ; Z / k \ell Z) \rightarrow \pi_{*-1}(X)$. The dual of the coinflation map is the inflation map $\eta: \pi_{*}(X ; Z / k Z) \rightarrow \pi_{*}(X ; Z / k \ell Z)$ and the Bocksteins $\beta$ : $\pi_{*}(X ; Z / k Z) \rightarrow \pi_{*-1}(X)$ are related by $\beta=\beta \cdot \eta: \pi_{*}(X ; Z / k \ell Z) \rightarrow \pi_{*-1}(X)$.

Theorem 5.2 The coreduction and coinflation maps combine to give a long cofibration sequence 


$$
P^{2}(k \ell) \stackrel{\bar{\eta}}{\rightarrow} P^{2}(\ell) \stackrel{\bar{\beta}}{\rightarrow} P^{3}(k) \stackrel{\bar{\eta}}{\rightarrow} P^{3}(k \ell) \stackrel{\bar{\rho}}{\rightarrow} P^{3}(\ell) \stackrel{\bar{\beta}}{\rightarrow} P^{4}(k) \stackrel{\bar{\eta}}{\rightarrow} \cdots
$$

The above dualizes in the pointed homotopy category to the long exact sequence of homotopy groups with coefficients.

\section{Universal coefficients and homotopy Bockstein spectral sequences}

Bocksteins, universal coefficient theorems, and Bockstein spectral sequences take the same form for homotopy groups with coefficients as they do for homology groups with coefficients. Furthermore, the Hurewicz maps map the homotopy versions to the homology versions.

The mod $p^{r}$ homotopy Bockstein exact sequence is the dual of the cofibration sequence

$$
S^{1} \stackrel{p^{r}}{\rightarrow} S^{1} \stackrel{\iota}{\rightarrow} P^{2}\left(p^{r}\right) \stackrel{q}{\rightarrow} S^{2} \stackrel{p^{r}}{\rightarrow} S^{2} \rightarrow \cdots
$$

that is

$$
\pi_{1}(X) \stackrel{p^{r}}{\longleftarrow} \pi_{1}(X) \stackrel{\beta}{\longleftarrow} \pi_{2}\left(X ; Z / p^{r} Z\right) \stackrel{\rho}{\longleftarrow} \pi_{2}(X) \stackrel{p^{r}}{\longleftarrow} \pi_{2}(X) \leftarrow \cdots
$$

This gives the homotopy version of the mod $p^{r}$ homology universal coefficient exact sequence

Theorem 6.1 (Universal coefficient exact sequence) There is a natural exact sequence

$$
0 \rightarrow \pi_{n}(X) \otimes Z / p^{r} Z \rightarrow \pi_{n}\left(X ; Z / p^{r} Z\right) \rightarrow \operatorname{Tor}\left(\pi_{n-1}(X), Z / p^{r} Z\right) \rightarrow 0
$$

Remark 6.2 The Hurewicz map gives a map of universal coefficient sequences.

Definition 6.3 Setting $r=1$ in the $\bmod p^{r}$ homotopy Bockstein exact sequence gives an exact couple [11-13] and hence a $\bmod p$ Bockstein spectral sequence with $E^{1}=\pi_{*}(X ; Z / p Z)$ and differentials $\beta^{r}: E^{r} \rightarrow E^{r}$ given by the relation $\beta^{r}[f]=$ $f \cdot \iota \cdot p^{1-r} \cdot q$, that is, the relation

$$
P^{n-1}(p) \stackrel{q}{\rightarrow} S^{n-1} \stackrel{p^{r-1}}{\longleftarrow} S^{n-1} \stackrel{\iota}{\rightarrow} P^{n}(p) \stackrel{f}{\rightarrow} X .
$$

Thus a class $[f]: P^{n}(p) \rightarrow X$ in $\pi_{n}(X ; Z / p Z)$ survives to $E^{r}$ if its restriction $f \cdot \iota$ to $S^{n-1}$ is divisible by $p^{r-1}, f \cdot \iota=g \cdot p^{r-1}$, and then $\beta^{r}[f]$ is represented by the composition

$$
g \cdot q: P^{n-1}(p) \rightarrow S^{n-1} \rightarrow X
$$

In order to further describe the differentials in the mod $p$ homotopy Bockstein spectral sequence we recall the coreduction maps $\bar{\eta}: P^{n}\left(p^{r}\right) \rightarrow P^{n}\left(p^{r+s}\right)$ which are 
degree $p^{s}$ on the bottom spheres $S^{n-1}$ and degree one on the top cells $e^{n}$. It follows that

Theorem 6.4 If $x$ generates a summand in $\pi_{n}(X)$ localized at $p$, then $x$ will correspond in the mod p homotopy Bockstein spectral sequence to

a) if $x$ has infinite order, there is an $n$-dimensional class $y=x \cdot q \in E^{1}=$ $\pi_{n}(X ; Z / p Z)$ which is an infinite cycle, that is, $\beta^{r} y=0$ for all $r \geq 1$

b) if $x$ has order $p^{r}$, then it extends to an $n+1$ dimensional class

$$
z: P^{n+1}\left(p^{r}\right) \rightarrow X, \quad z \cdot \iota=x
$$

and thus there are two classes

$$
y=x \cdot q: P^{n}(p) \rightarrow S^{n} \rightarrow X
$$

in $E^{1}=\pi_{n}(X ; Z / p Z)$ in dimension $n$ and

$$
w=z \cdot \bar{\rho}: P^{n+1}(p) \stackrel{\bar{\rho}}{\rightarrow} P^{n+1}\left(p^{r}\right) \stackrel{z}{\rightarrow} X
$$

in $E^{1}=\pi_{n+1}(X ; Z / p Z)$ in dimension $n+1$. Both $y$ and $w$ survive to $E^{r}$ with $\beta^{r} w=y$.

In summary, in dimension $n$ of integral homotopy, summands of infinite order are represented by $n$ dimensional infinite cycles and summands of order $p^{r}$ are detected by a nonzero $r$-th Bockstein differential sending an $n+1$ dimensional class to an $n$ dimensional class.

Remark 6.5 The long exact homology sequences associated to the short exact coefficient sequences $0 \rightarrow Z \stackrel{p^{r}}{\rightarrow} Z \rightarrow Z / p^{r} Z \rightarrow 0$ yield long exact mod $p^{r}$ homology Bockstein sequences. The Hurewicz maps yield commutative diagrams which send the homotopy Bockstein exact sequences to the homology Bockstein exact sequences. Setting $r=1$ yields the mod $p$ homology Bockstein exact couple and the mod $p$ homology Bockstein spectral sequence. The Hurewicz maps yield morphisms of Bockstein exact couples and hence of Bockstein spectral sequences. The differentials in the homology Bockstein spectral sequence have a similar description to those in the homotopy Bockstein spectral sequence [2].

Remark 6.6 Using the methods of [3], the $r$-th term of the mod $p$ homotopy Bockstein spectral sequence can be explicitly identified $E^{r}=$ image $p^{r-1}: \pi_{*}\left(X ; Z / p^{r} Z\right) \rightarrow$ $\pi_{*}\left(X ; Z / p^{r} Z\right)$.

\section{Graded Lie algebras}

The definition of a graded Lie algebra has a complication when 2 is not a unit in the ground ring. It is necessary to add another operation to the usual bilinear Lie bracket 
operation. Namely, one must add a quadratic operation $x \mapsto x^{2}$ which is defined on odd degree classes. If 2 is a unit in the ground ring, then this squaring operation may be omitted since $x^{2}=\frac{1}{2}[x, x]$ for odd degree $x$. More precisely,

Definition 7.1 A graded Lie algebra $L$ is a graded $R$ module together with two operations:

(1) bilinear pairings called Lie brackets

$$
[\quad, \quad]: L_{m} \otimes L_{n} \rightarrow L_{m+n}, x \otimes y \mapsto[x, y]
$$

(2) and a quadratic operation called squaring defined on odd degree classes

$$
()^{2}: L_{k} \rightarrow L_{2 k}, \quad x \mapsto x^{2}
$$

with $k$ odd. The quadratic requirement is expressed in the identities

$$
\begin{gathered}
(a x)^{2}=a^{2} x^{2}, \quad(x+y)^{2}=x^{2}+y^{2}+[x, y] \text { for all scalars } a \\
\text { and all } x \text { and } y \text { of equal odd degree. }
\end{gathered}
$$

These operations must satisfy the identities

(1) anti-commutativity:

$$
[x, y]=-(-1)^{\operatorname{deg}(x) \operatorname{deg}(y)}[y, x] \text { for all } x, y,
$$

(2) Jacobi identity:

$$
[x,[y, z]]=[[x, y], z]+(-1)^{\operatorname{deg}(x) \operatorname{deg}(y)}[y,[x, z]] \text { for all } x, y, z,
$$

(3) double vanishing:

$$
[x, x]=0 \text { for all } x \text { of even degree, }
$$

(4) divisibility and triple vanishing:

$$
2 x^{2}=[x, x], \quad\left[x, x^{2}\right]=0 \quad \text { for all } x \text { of odd degree. }
$$

(5)

$$
\left[y, x^{2}\right]=[[y, x], x]
$$

for all $y$ and for all $x$ of odd degree. 
Remark 7.2 The first example of a graded Lie algebra is a graded associative algebra $A$ with the Lie bracket

$$
[a, b]=a b-(-1)^{\operatorname{deg}(a) \operatorname{deg}(b)} b a
$$

and the squaring operation $c^{2}$ for $c$ of odd degree. The above identities are all valid. If a graded Lie algebra is to embed in its universal enveloping algebra as a split summand, we must have all of the above identities and the squaring operation.

Definition 7.3 If a graded Lie algebra $L$ as above has a linear degree -1 map $d: L \rightarrow L$ such that

(1) it has square $0, d^{2}=0$,

(2) it is a derivation, $d[x, y]=[d x, y]+(-1)^{\operatorname{deg}(x)}[x, d y], \quad d\left(z^{2}\right)=[d z, z]$ if $z$ has odd degree,

then it is called a differential Lie algebra.

Of course, morphisms of graded Lie algebras are required to preserve the product and squaring structures. Morphisms of differential graded Lie algebras must also preserve the differential.

Remark 7.4 If 2 is a unit in the ground ring, then the squaring operation may be defined in terms of the Lie bracket, that is, $x^{2}=\frac{1}{2}[x, x]$ for $x$ of odd degree. In the axioms for a graded Lie algebra, we may omit all reference to the squaring operation and add the requirement that $[x,[x, x]]=0$ for all $x$ of odd degree.

If $x$ has even degree, then anti-commutavity yields $2[x, x]=0$. If $x$ has odd degree, the Jacobi identity yields that $3[x,[x, x]]=0$. Thus, if 2 and 3 are units in the ground ring, we may omit all reference to the vanishing conditions on double and triple products.

Let $G$ be a grouplike space. If we invert the primes 2 and 3, the Samelson product

$$
[\quad, \quad]: \pi_{*}(G) \otimes \pi_{*}(G) \rightarrow \pi_{*}(G)
$$

makes the integral homotopy groups into a graded Lie algebra. But, if we do not invert 2, the nonexistence of elements of Hopf invariant one [1] prevents both the existence of a squaring operation and the double vanishing condition.

That is, if there existed a homotopy squaring operation such that $2 x^{2}=[x, x]$ for odd dimensional $x$, then the class $\iota_{2 n+1}^{2} \in H_{*}\left(\Omega S^{2 n+2} ; Z\right)$ would be spherical and Serre's proof would show that there is a homotopy equivalence

$$
S^{2 n+1} \times \Omega S^{4 n+3} \stackrel{\simeq}{\rightarrow} \Omega S^{2 n+2}
$$

and, without any localization away from $2, S^{2 n+1}$ would be an H-space. But this is impossible unless $n=0,1$, or 3 .

If $[x, x]=0$ were always valid for even dimensional $x$, then the adjoint Whitehead product $[z, z]_{w}=0$ would vanish for odd dimensional $z$. If $\iota_{2 n+1}: S^{2 n+1} \rightarrow S^{2 n+1}$ 
is the identity map, then the Whitehead product $\left[\iota_{2 n+1}, \iota_{2 n+1}\right]_{w}=0$ and again $S^{2 n+1}$ would be an $\mathrm{H}$-space.

If we do not invert 3 , then the nonexistence of elements of odd Hopf invariant one $[10,24]$ prevents the triple vanishing condition. That is, if $[x,[x, x]]=0$ for all odd dimensional $x$, the vanishing Whitehead products $\left[\iota_{2 n},\left[\iota_{2 n}, \iota_{2 n}\right]_{w}\right]_{w}=0$ would contradict $\bmod 3$ Hopf invariant one unless $n=1$.

In a different way, the primes 2 and 3 cause problems with the Samelson products in homotopy groups with 2 primary or 3 primary coefficients. We cannot even define the Samelson products in mod 2 homotopy, much less the squaring maps. In mod $2^{r}$ homotopy with $r \geq 2$ we can define the Samelson products but probably not the squaring operations. More important, the Lie identities may fail.

In mod 3 homotopy, the Jacobi identity may fail. But the Bockstein spectral sequence, from the second term onwards, restores the Jacobi identity and, from the third term onwards, the triple vanishing condition is restored. In dimensions $\geq 3$, the Jacobi identity is valid in mod $3^{r}$ homotopy for $r \geq 2$. It is not known if the triple vanishing condition is valid in mod $3^{r}$ homotopy when $r \geq 3$.

In this paper we will focus entirely on Samelson products in homotopy groups with coefficients and leave the classical products in integral homotopy as an easier case to be worked out by the reader.

\section{Samelson products and differential Lie identities}

Samelson products in the homotopy groups of grouplike spaces are graded Lie structures for which the Hurewicz map is a representation into the Lie structure of the Pontrjagin ring.

Let $G$ be a grouplike space and let [ , ] : $G \times G \rightarrow G$ be the commutator map $[x, y]=x y x^{-1} y^{-1}$. This factors uniquely up to homotopy as

$$
G \times G \rightarrow G \wedge G \stackrel{[, \quad]}{\longrightarrow} G
$$

Let $p$ be a prime and suppose $p^{r}>2$.

Definition 8.1 If $f: P^{n}\left(p^{r}\right) \rightarrow G$ and $g: P^{m}\left(p^{r}\right) \rightarrow G$ are two homotopy classes, then the internal Samelson product $[f, g]$ is represented by the composition

$$
P^{n+m}\left(p^{r}\right) \stackrel{\Delta}{\rightarrow} P^{n}\left(p^{r}\right) \wedge P^{m}\left(p^{r}\right) \stackrel{f \wedge g}{\longrightarrow} G \wedge G \stackrel{[, \quad]}{\longrightarrow} G .
$$

Note: At odd primes, the choice of $\Delta$ is unique up to homotopy. If $p=2$, the choice is not unique but at least we can choose one to make a definition.

Theorem 8.2 The Samelson product defines bilinear pairings

$$
[\quad, \quad]: \pi_{n}\left(G: Z / p^{r} Z\right) \otimes \pi_{m}\left(G ; Z / p^{r} Z\right) \rightarrow \pi_{m+n}\left(G ; Z / p^{r} Z\right)
$$

which are natural with respect to H maps and which give the structure of a graded Lie algebra if $p>3$, that is, 
a) if $p$ is odd, it is bilinear, $[f+g, h]=[f, h]+[g, h]$ and anti-commutative, $[f, h]=-(-1)^{\operatorname{deg}(f) \operatorname{deg}(h)}[h, f]$.

b) if $p>3$, the Jacobi identity is valid, $[f,[g, h]]=[[f, g], h]$ $+(-1)^{\operatorname{deg}(f)} \operatorname{deg}(g)[g,[f, h]]$.

Remark 8.3 The Lie identities for Samelson products are consequences of the so-called Lie identities for elements in groups [23,27,28]. The failure of the Jacobi identity when $p=3$ is a consequence of the failure of the coassociativity of the maps $\Delta$.

Remark 8.4 If 2 is a unit, the Lie identities imply that $[f, f]=0$ for all even degree $f$ but not for elements of odd degree. Thus, the free graded Lie algebra $L(f)$ is the vector space $<f>$ if $f$ has even degree. But $L(f)$ is the rank two vector space $<f,[f, f]>$ if $f$ has odd degree. If 3 is a unit, then $[f,[f, f]]=0$ for all odd degree $f$ and hence for all $f$.

Theorem 8.5 The Samelson product defines a bilinear pairing of homotopy Bockstein spectral sequences $[]:, E^{r} \otimes E^{r} \rightarrow E^{r}$. In particular, the differentials $\beta^{r}$ are derivations, $\beta^{r}[f, g]=\left[\beta^{r} f, g\right]+(-1)^{\operatorname{deg}(f)}\left[f, \beta^{r} g\right]$. Furthermore, the terms $E^{r}$ are differential graded Lie algebras whenever $p>3$ or $p=3, r \geq 3$.

For proofs of the above theorems, see $[16,19]$. In particular, the treatment of products in a spectral sequence requires the introduction of the spectral systems of CartanEilenberg [3].

Remark 8.6 The reduction maps $\rho: \pi_{*}\left(G ; Z / p^{r+s} Z\right) \rightarrow \pi_{*}\left(G ; Z / p^{r}\right)$ are morphisms of Lie structures and the inflation maps $\eta: \pi_{*}\left(G ; Z / p^{r} Z\right) \rightarrow$ $\pi_{*}\left(G ; Z / p^{r+s} Z\right)$ are morphisms up to a factor, that is, $\rho[f, g]=[\rho f, \rho g], p^{s} \eta[f, g]$ $=[\eta f, \eta g]$.

Remark 8.7 The Hurewicz map $\phi: \pi_{*}\left(G ; Z / p^{r} Z\right) \rightarrow H_{*}\left(G ; Z / p^{r} Z\right)$ is a morphism of Lie structures, that is,

$$
\phi[f, g]=[\phi f, \phi g]=(\phi f)(\phi g)-(-1)^{\operatorname{deg}(f) \operatorname{deg}(g)}(\phi g)(\phi f)
$$

whenever $p$ is an odd prime. Similarly, $\phi$ is a morphism of the differential graded Lie algebras in the mod $p$ Bockstein spectral sequences.

\section{Compressions of Samelson products}

In applications it is sometimes important to compress Samelson products into the fibres of loop maps. And it is vital that the Lie identities such as anti-commutativity and the Jacobi identity are preserved.

In this section we consider Samelson product versions of the relative cup products

$$
H^{n}(X, A) \otimes H^{m}(X, B) \rightarrow H^{n+m}(X, A \cup B)
$$

In this case, explicit formulas with cochains show that

$$
x \in H^{n}(X, A), y \in H^{m}(X, B) \Longrightarrow x \cup y \in H^{n+m}(X, A \cup B)
$$


and that the usual associative and commutative laws hold for these relative cup products. In the case of relative Samelson products we do not have the advantage of cochains and we must find other methods to verify the identities for relative Samelson products.

One feature shared by relative Samelson products and relative cup products is that we often do not distinguish in the notation between the absolute and relative products.

If $N$ is a normal subgroup of $G$, then the commutator factors as

$$
[,]: N \times G \rightarrow N \subseteq G, \quad x \in N, y \in G \Longrightarrow[x, y]=x y x^{-1} y^{-1} \in N
$$

This has a Samelson product version. Let $p$ be an odd prime and let $F \stackrel{\iota}{\rightarrow} E \stackrel{q}{\rightarrow} B$ be a fibration sequence.

Theorem 9.1 There are bilinear pairings

$$
\begin{aligned}
{[,]_{r}=[,] } & : \pi_{n}\left(\Omega F ; Z / p^{r} Z\right) \otimes \pi_{m}\left(\Omega E ; Z / p^{r} Z\right) \rightarrow \pi_{n+m}\left(\Omega F ; Z / p^{r} Z\right) \\
& \times \in \pi_{n}\left(\Omega F ; Z / p^{r}\right), y \in \pi_{m}\left(\Omega E ; Z / p^{r} Z\right) \\
& \Longrightarrow[x, y]=[x . y]_{r} \in \pi_{n+m}\left(\Omega F ; Z / p^{r} Z\right)
\end{aligned}
$$

called relative Samelson products. The product can also be done in the other order, that is, $[y, x]_{r}$.

a) The relative products are natural with respect to maps of fibration sequences

b) Samelson products and relative Samelson products are compatible, that is, the following diagrams commute

$$
\begin{array}{ccc}
\pi_{n}\left(\Omega F ; Z / p^{r} Z\right) \otimes \pi_{m}\left(\Omega F ; Z / p^{r} Z\right) \stackrel{[, \quad]}{\longrightarrow} \pi_{n+m}\left(\Omega F, Z / p^{r} Z\right) \\
\downarrow 1 \otimes \iota_{*} & \downarrow 1 \\
\pi_{n}\left(\Omega F, Z / p^{r} Z\right) \otimes \pi_{m}\left(\Omega E ; Z / p^{r} Z\right) \stackrel{[\quad, \quad] r}{\longrightarrow} \pi_{n+m}\left(\Omega F ; Z / p^{r} Z\right) \\
\downarrow \iota_{*} \otimes 1 & \downarrow \iota_{*} \\
\pi_{n}\left(\Omega E ; Z / p^{r} Z\right) \otimes \pi_{m}\left(\Omega E ; Z / p^{r} Z\right) \stackrel{[\quad, \quad]}{\longrightarrow} & \pi_{n+m}\left(\Omega E ; Z / p^{r} Z\right)
\end{array}
$$

c) The Lie identities hold, that is,

$$
[x, y]=-(-1)^{\operatorname{deg}(x) \operatorname{deg}(y)}[y, x]
$$

whenever $x$ and $y$ are in the union of $\pi_{*}\left(\Omega F ; Z / p^{r} Z\right)$ and $\pi_{*}\left(\Omega E ; Z / p^{r} Z\right)$.

$$
[x,[y, z]]=[[x, y], z]+(-1)^{\operatorname{deg}(x) \operatorname{deg}(y)}[y,[x, z]]
$$

whenever $p>3$ and $x, y$, and $z$ are in the union of $\pi_{*}\left(\Omega F ; Z / p^{r} Z\right)$ and $\pi_{*}\left(\Omega E ; Z / p^{r} Z\right)$. 
d) The relative Samelson products give pairings of the mod p homotopy Bockstein spectral sequences with the differentials being derivations, $\beta^{r}[x, y]=\left[\beta^{r} x, y\right]+$ $(-1)^{\operatorname{deg}(x)}\left[x, \beta^{r} y\right]$. The pairings of mod p homotopy Bockstein spectral sequences satisfy the Lie identities in $c$ ). Even if $p=3$, the Jacobi identity is valid if $r \geq 2$ and the triple vanishing condition is valid if $r \geq 3$.

It is trivial that the Samelson products factor through the homotopy of the loops on the fibre when one of the factors comes from the homotopy of the loops on the fibre. What is nontrivial is that the above Lie identities in c) and the derivation formulas in d) are valid.

Remark 9.2 The above theorem can be proved with the use of Kan's simplicial group model $\mathrm{K}(\mathrm{X})[9,14]$ for the loop space of $X$. Indeed, if $K(q): K(E) \rightarrow K(B)$ is the surjective homomorphism of group models for $\Omega q: \Omega E \rightarrow \Omega B$, then the kernel $K$ of $K(q)$ is a normal subgroup which is a model for $\Omega F$. Hence, the appropriate commutators factor through $K, \quad[\quad, \quad]: K \times K(E) \rightarrow K$. It follows that the group theoretic identities used for the Samelson product apply without change to give the Lie identities and the derivation formulas for the relative Samelson products.

When $p>3$, the relative Samelson products give $\pi_{*}\left(\Omega F ; Z / p^{r} Z\right) \rightarrow$ $\pi_{*}\left(\Omega E ; Z / p^{r} Z\right)$ the structure of an extended Lie ideal.

Definition 9.3 Let $L^{\prime} \rightarrow L$ be a morphism of graded Lie algebras. We call $L^{\prime}$ an extended ideal of $L$ if there are two bilinear pairings (called Lie brackets):

$$
\begin{aligned}
& {[\quad, \quad]: L^{\prime} \times L \rightarrow L^{\prime}} \\
& {[\quad, \quad]: L \times L^{\prime} \rightarrow L^{\prime},}
\end{aligned}
$$

such that

(1) the diagram of Lie brackets commutes

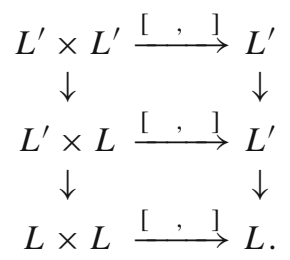

(2) for all $x, y$, and $z$ in the union of $L^{\prime}$ and $L$,

$$
\begin{gathered}
{[x, y]=-(-1)^{\operatorname{deg}(x) \cdot \operatorname{deg}(y)}[y, x]} \\
{[x,[y, z]]=[[x, y], z]+(-1)^{\operatorname{deg}(x) \cdot \operatorname{deg}(y)}[y,[x, z]] .}
\end{gathered}
$$

(3) if $x$ has odd degree and either $x$ or $y$ is in $L^{\prime}$, then $\left[y, x^{2}\right]=[[y, x], x]$. 
Definition 9.4 An extended differential ideal $L^{\prime} \rightarrow L$ is a morphism of differential graded Lie algebras which is an extended ideal and such that the differential $d$ is a derivation in the sense that, for all $x$ and $y$ in the union of $L^{\prime}$ and $L$,

$$
d[x, y]=[d x, y]+(-1)^{\operatorname{deg}(x)}[x, d y] .
$$

\section{H-based compressions of Samelson products}

If $N$ is a normal subgroup of a group $G$ with the quotient $G / N$ abelian, then there is a factorization of the commutator

$$
[\quad, \quad]: G \times G \rightarrow N \subseteq G, \quad x \in G, y \in G \Longrightarrow[x, y]=x y x^{-1} y^{-1} \in N
$$

The Samelson product version of this occurs for a fibration $F \rightarrow E \rightarrow B$ when the base $B$ is an $\mathrm{H}$-space. In this case, the loop space $\Omega B$ is homotopy commutative.

Theorem 10.1 Let $B$ be an $H$-space with multiplication $\mu: B \times B \rightarrow B$. There are bilinear pairings

$$
\begin{aligned}
{[,]_{\mu}=[, \quad]: } & \pi_{n}\left(\Omega E ; Z / p^{r} Z\right) \otimes \pi_{m}\left(\Omega E ; Z / p^{r} Z\right) \rightarrow \pi_{n+m}\left(\Omega F, ; Z / p^{r} Z\right) \\
x & \in \pi_{n}\left(\Omega E ; Z / p^{r}\right), \quad y \in \pi_{m}\left(\Omega E ; Z / p^{r} Z\right) \\
& \Longrightarrow[x, y]=[x, y]_{\mu} \in \pi_{n+m}\left(\Omega F ; Z / p^{r} Z\right)
\end{aligned}
$$

These are called H-based Samelson products.

a) The H-based products are natural with respect to maps of fibration sequences covering H-maps on the bases.

b) Samelson products, relative Samelson products, and H-based Samelson products are compatible, that is, the following diagrams commute

$$
\begin{array}{cccc}
\pi_{n}\left(\Omega F ; Z / p^{r} Z\right) \otimes \pi_{m}\left(\Omega F ; Z / p^{r} Z\right) \stackrel{[, \quad]}{\longrightarrow} & \pi_{n+m}\left(\Omega F ; Z / p^{r} Z\right) \\
\downarrow 1 \otimes \iota_{*} & \downarrow 1 \\
\pi_{n}\left(\Omega F ; Z / p^{r} Z\right) \otimes \pi_{m}\left(\Omega E ; Z / p^{r} Z\right) \stackrel{[\quad, \quad]_{r}}{\longrightarrow} & \pi_{n+m}\left(\Omega F ; Z / p^{r} Z\right) \\
\downarrow \iota_{*} \otimes 1 & \downarrow 1 \\
\pi_{n}\left(\Omega E ; Z / p^{r} Z\right) \otimes \pi_{m}\left(\Omega E ; Z / p^{r} Z\right) \stackrel{[\quad, \quad]_{\mu}}{\longrightarrow} & \pi_{n+m}\left(\Omega F ; Z / p^{r} Z\right) \\
\downarrow 1 & \downarrow & \downarrow \iota_{*} \\
\pi_{n}\left(\Omega E ; Z / p^{r} Z\right) \otimes \pi_{m}\left(\Omega E ; Z / p^{r} Z\right) \stackrel{[\quad, \quad]}{\longrightarrow} & \pi_{n+m}\left(\Omega E ; Z / p^{r} Z\right)
\end{array}
$$

c) The Lie identities hold, that is, if $\mu \cdot T$ is the opposite multiplication to $\mu$, $\mu \cdot T(a, b)=\mu(b, a)$, then

$$
[x, y]_{\mu}=-(-1)^{\operatorname{deg}(x) \operatorname{deg}(y)}[y, x]_{\mu \cdot T}
$$


whenever $x$ and $y$ are in the union of $\pi_{*}\left(\Omega F ; Z / p^{r} Z\right)$ and $\pi_{*}\left(\Omega E ; Z / p^{r} Z\right)$.

$$
\left[x,[y, z]_{\mu}\right]_{r}=\left[[x, y]_{\mu}, z\right]_{r}+(-1)^{\operatorname{deg}(x) \operatorname{deg}(y)}\left[y,[x, z]_{\mu}\right]_{r}
$$

whenever $p>3$ and $x, y$, and $z$ are in the union of $\pi_{*}\left(\Omega F ; Z / p^{r} Z\right)$ and $\pi_{*}\left(\Omega E ; Z / p^{r} Z\right)$.

d) The H-based Samelson products give pairings of the mod $p$ homotopy Bockstein spectral sequences with the differentials being derivations, $\beta^{r}[x, y]_{\mu}=$ $\left[\beta^{r} x, y\right]_{\mu}+(-1)^{\operatorname{deg}(x)}\left[x, \beta^{r} y\right]_{\mu}$. The pairings of mod p homotopy Bockstein spectral sequences satisfy the Lie identities in $c$ ). Even if $p=3$, the Jacobi identity is valid if $r \geq 2$ and the triple vanishing condition is valid if $r \geq 3$.

Remark 10.2 If $B$ has a homotopy commutative multiplication, then $[y, x]_{\mu \cdot T}=[y, x]$ and we have the true Lie anti-commutativity in c) above.

Remark 10.3 Since any strictly commutative topological group is a generalized Eilenberg-MacLane space [14], there is no possibility of using commutative group models to prove the Lie identities for general H-based Samelson products.

The H-based Samelson products are an example of strong extended ideals.

Definition 10.4 Let $L^{\prime} \rightarrow L$ be a morphism of graded Lie algebras which is an extended ideal. We call $L^{\prime}$ a strong extended ideal of $L$ if there is a bilinear pairing (called a Lie bracket):

$$
[\quad, \quad]: L \times L \rightarrow L^{\prime}
$$

and a squaring operation

$$
()^{2}: L_{2 n+1} \rightarrow L_{4 n+2}^{\prime}
$$

such that

(1) the diagram of Lie brackets commutes

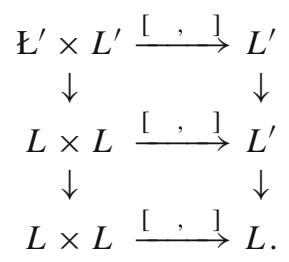

(2) the diagram of squaring operations commutes 


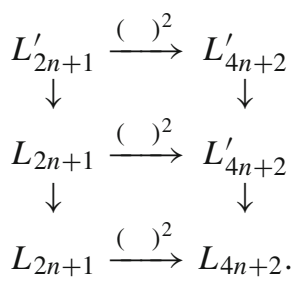

(3) for all odd degree $x$ in $L$,

$$
2 x^{2}=[x, x] \in L^{\prime}, \quad\left[y, x^{2}\right]=[[y, x], x] \in L^{\prime}
$$

(4) the squaring operation is quadratic, for all $x$ and $y$ of the same odd degree in $L^{\prime}$ and for all scalars $a$,

$$
(a x)^{2}=a^{2} x^{2}, \quad(x+y)^{2}=x^{2}+[x, y]+y^{2} .
$$

Definition 10.5 A strong extended differential ideal is a differential ideal $L^{\prime} \rightarrow L$ such that the differential $d$ satisfies $d\left(x^{2}\right)=[d x, x] \in L^{\prime}$ for all odd degree $x$ in $L$.

\section{Exercises}

(1) If $\iota: K \rightarrow L$ is the inclusion of a differential ideal, then $\iota_{*}: H K \rightarrow H L$ is an extended ideal.

(2) If, in addition, $L / K$ is abelian, then $\iota_{*}: H K \rightarrow H L$ is a strong extended ideal.

(3) Connected differential graded Lie algebras form a model category for the rational homotopy theory of simply connected spaces [21]. In particular, if $L_{X}$ is the Lie algebra model for $X$, then $\pi_{*}(\Omega X) \otimes Q \simeq H L_{X}$ as graded Lie algebras. Show that exercises 1 and 2 prove the existence and Lie identities for relative Samelson products and for H-based Samelson products for rational homotopy theory.

\section{Universal models for relative Samelson products}

In this section and the next, we indicate how universal models show that the existence and the identities for a natural absolute Samelson product in $\pi_{*}\left(\Omega E ; Z / p^{r} Z\right)$ implies the existence and the identities for the relative Samelson products and the H-based Samelson products introduced in the previous section. The well defined existence and the identities are both consequences of the Hilton-Milnor theorem.

Recall the adjunction maps $e: \Sigma \Omega X \rightarrow X, \quad e\langle t, \omega\rangle=\omega(t)$ and $\Sigma: X \rightarrow$ $\Omega \Sigma X, \quad \Sigma(x)(t)=\langle t, x\rangle=\omega(t), \quad \Sigma(x)=\omega=\langle, x\rangle$.

Definition 11.1 If $f: Y \rightarrow \Omega X$ is a map, then

$$
\bar{f}: \Sigma Y \stackrel{\Sigma f}{\rightarrow} \Sigma \Omega X \stackrel{e}{\rightarrow} X
$$

is the adjoint and

$$
\Omega \bar{f}: \Omega \Sigma Y \rightarrow \Omega X
$$


is the multiplicative extension. The multiplicative extension is a loop map which extends $f$ in the sense that the composition $\Omega \bar{f} \cdot \Sigma: Y \stackrel{\Sigma}{\rightarrow} \Omega \Sigma Y \stackrel{\Omega \bar{f}}{\rightarrow} \Omega X$ is $f$.

The classical universal model for absolute Samelson products is given as follows:

Suppose $f: P^{n}\left(p^{r}\right) \rightarrow \Omega E$ and $g: P^{m}\left(p^{r}\right) \rightarrow \Omega E$ are two maps. These define a map $f \vee g: P^{n}\left(p^{r}\right) \vee P^{m}\left(p^{r}\right) \rightarrow \Omega E$ which has multiplicative extension $\Phi: \Omega \Sigma\left(P^{n}\left(p^{r}\right) \vee P^{m}\left(p^{r}\right)\right) \rightarrow \Omega E$. Denote the standard maps by $\iota_{n}: P^{n}\left(p^{r}\right) \rightarrow$ $\Omega \Sigma\left(P^{n}\left(p^{r}\right) \vee P^{m}\left(p^{r}\right)\right)$ and $\iota_{m}: P^{m}\left(p^{r}\right) \rightarrow \Omega \Sigma\left(P^{n}\left(p^{r}\right) \vee P^{m}\left(p^{r}\right)\right)$.

Hence,

$$
\Phi_{*} \iota_{n}=f, \quad \Phi_{*} \iota_{m}=g, \text { and } \quad \Phi_{*}\left(\left[\iota_{n}, \iota_{m}\right]\right)=[f, g]
$$

Thus the Samelson product $\left[\iota_{n}, \iota_{m}\right]$ in the universal model $\Omega \Sigma\left(P^{n}\left(p^{r}\right) \vee P^{m}\left(p^{r}\right)\right)$ determines the Samelson product in $\Omega E$ and could be used to give a natural definition if we had not already done so.

Suppose now that $\Omega F \stackrel{\Omega \iota}{\longrightarrow} \Omega E \stackrel{\Omega q}{\longrightarrow} \Omega B$ is the loops on a fibration sequence. If $f: P^{n}\left(p^{r}\right) \rightarrow \Omega F$ and $g: P^{m}\left(p^{r}\right) \rightarrow \Omega E$ are two maps, we construct a universal model for the relative Samelson product $[f, g]: P^{n+m}\left(p^{r}\right) \rightarrow \Omega F$ as follows.

If $h: X \rightarrow Y$ is any map, it can be replaced up to homotopy equivalence by a fibration. That is, there is a natural factorization

$$
h=\iota \cdot \tilde{h}: X \rightarrow \tilde{X} \rightarrow Y
$$

where $\iota$ is a homotopy equivalence and a cofibration and $\tilde{h}$ is a fibration.

Consider the projection map $\pi: \Sigma\left(P^{n}\left(p^{r}\right) \vee P^{m}\left(p^{r}\right)\right) \rightarrow \Sigma P^{m}\left(p^{r}\right)$ and replace it by a fibration $\tilde{\pi}$ as above:

$$
\Sigma\left(P^{n}\left(p^{r}\right) \vee P^{m}\left(p^{r}\right)\right) \stackrel{\iota}{\rightarrow} E^{n, m} \stackrel{\tilde{\pi}}{\rightarrow} \Sigma P^{m}\left(p^{r}\right)
$$

Let $F^{n, m} \stackrel{\iota}{\rightarrow} E^{n, m} \stackrel{\tilde{\pi}}{\rightarrow} \Sigma P^{m}\left(p^{r}\right)$ be the fibration sequence. The adjoint maps define a map $\bar{f} \vee \bar{g}: \Sigma\left(P^{n}\left(p^{r}\right) \vee P^{m}\left(p^{r}\right)\right) \rightarrow E$ with $\bar{f}: \Sigma P^{n}\left(p^{r}\right) \rightarrow F$. Up to fibre homotopy, there is a unique extension to a map $\tilde{\Phi}: E^{n, m} \rightarrow E, \tilde{\Phi} \cdot \iota=\bar{f} \vee \bar{g}$, which makes the following diagram commutative

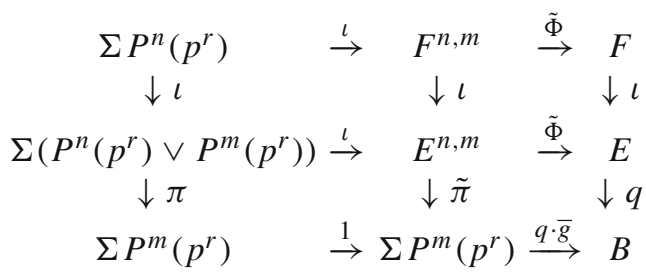

The Samelson product $\left[\iota_{n}, \iota_{m}\right]$ in $\Omega E^{n, m}$ actually lies in the homotopy of $\Omega F^{n, m}$. Denote this element by $\left[\iota_{n}, \iota_{m}\right]_{r}$,

$$
\Omega \iota_{*}\left[\iota_{n}, \iota_{m}\right]_{r}=\left[\iota_{n}, \iota_{m}\right] .
$$


The Hilton-Milnor theorem [19,28],

$$
\Omega \Sigma(X \vee Y) \simeq \Omega \Sigma X \times \Omega\left(\Sigma \bigvee_{k \geq 0} X^{k} \wedge Y\right)
$$

implies that the fibration $\Omega F^{n, m} \rightarrow \Omega E^{n, m} \stackrel{\Omega \tilde{\pi}}{\longrightarrow} \Omega \Sigma P^{m}\left(p^{r}\right)$ has a section. Hence, there is a unique choice of $\left[\iota_{n}, \iota_{m}\right]_{r}$ in $\Omega F^{n, m}$ and, if $\Phi=\Omega \tilde{\Phi}$, then

$$
\Phi_{*}\left[\iota_{n}, \iota_{m}\right]_{r}=[f, g]_{r}
$$

is a well defined definition of the relative Samelson product in the homotopy of $\Omega F$.

The validity of bilinearity and the Lie identities in the homotopy of $\Omega \Sigma\left(P^{n}\left(p^{r}\right) \vee\right.$ $\left.P^{m}\left(p^{r}\right)\right)$ and $\Omega \Sigma\left(P^{n}\left(p^{r}\right) \vee P^{m}\left(p^{r}\right) \vee P^{q}\left(p^{r}\right)\right)$ imply the same validity for all relative Samelson products. With more work, the same is true for mod $p$ homotopy Bockstein spectral sequences. These proofs are similar to and slightly simpler than those for the H-based Samelson products. We refer to [19] for details.

\section{Universal models for H-based Samelson products}

In this section we exhibit a universal model for $\mathrm{H}$-based products. One complication is that, in the universal model for H-based products, the base is not the loops on an $\mathrm{H}$-space. Nonetheless, the model works to give a well defined definition of H-based products and the Lie identities will be valid for primes greater than 3 .

Suppose that $\Omega F \stackrel{\Omega l}{\longrightarrow} \Omega E \stackrel{\Omega q}{\longrightarrow} \Omega B$ is the loops on a fibration sequence where $B$ is an H-space with multiplication $\mu: B \times B \rightarrow B$. If $f: P^{n}\left(p^{r}\right) \rightarrow \Omega E$ and $g: P^{m}\left(p^{r}\right) \rightarrow \Omega E$ are two maps, we construct a universal model for the H-based Samelson product $[f, g]_{\mu}: P^{n+m}\left(p^{r}\right) \rightarrow \Omega F$ as follows.

Replace the map $\pi: \Sigma\left(P^{n}\left(p^{r}\right) \vee P^{m}\left(p^{r}\right)\right) \rightarrow \Sigma P^{m}\left(p^{r}\right) \times \Sigma P^{m}\left(p^{r}\right)$ by a homotopy equivalent fibration $\tilde{\pi}$ as in the previous section, that is, factor the map into the composition of a cofibration homotopy equivalence followed by a fibration:

$$
\Sigma\left(P^{n}\left(p^{r}\right) \vee P^{m}\left(p^{r}\right)\right) \stackrel{\iota}{\rightarrow} \bar{E}^{n, m} \stackrel{\tilde{\pi}}{\rightarrow} \Sigma P^{n}\left(p^{r}\right) \times \Sigma P^{m}\left(p^{r}\right) .
$$

Let

$$
\bar{F}^{n, m} \stackrel{\iota}{\rightarrow} \bar{E}^{n, m} \stackrel{\tilde{\pi}}{\rightarrow} \Sigma P^{n}\left(p^{r}\right) \times \Sigma P^{m}\left(p^{r}\right)
$$

be the resulting fibration sequence.

The adjoint maps define a map $\bar{f} \vee \bar{g}: \Sigma\left(P^{n}\left(p^{r}\right) \vee P^{m}\left(p^{r}\right)\right) \rightarrow E$. The multiplication $\mu$ defines an extension $\tilde{\mu}$ of the map $\left.q \cdot(\bar{f} \vee \bar{g}): \Sigma P^{n}\left(p^{r}\right) \vee \Sigma P^{m}\left(p^{r}\right)\right) \rightarrow E \rightarrow B$ to the product, that is,

$$
\tilde{\mu}: \Sigma P^{n}\left(p^{r}\right) \times \Sigma P^{m}\left(p^{r}\right) \stackrel{q \bar{f} \times q \bar{g}}{\longrightarrow} B \times B \stackrel{\mu}{\rightarrow} B
$$


The diagram below commutes:

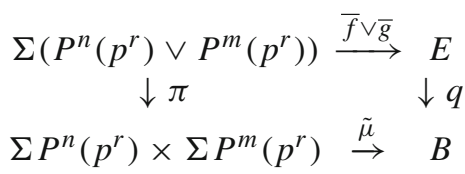

Up to fibre homotopy, there is a unique extension of $\bar{f} \vee \bar{g}$ to a map $\tilde{\Psi}: \underline{E}^{n, m} \rightarrow E$ which makes the following diagram commute:

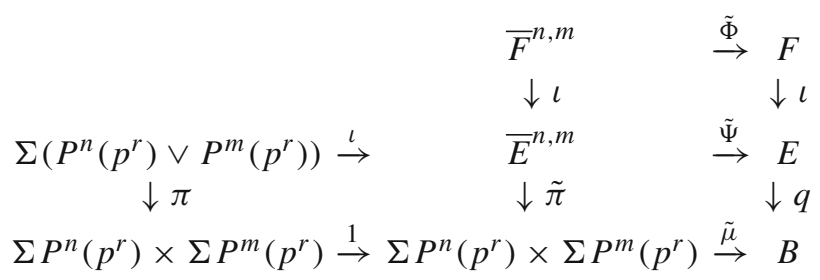

The Samelson product $\left[\iota_{n}, \iota_{m}\right]$ in $\Omega \bar{E}^{n, m}$ actually lies in the homotopy of $\Omega \bar{F}^{n, m}$. Denote this element by $\left[\iota_{n}, \iota_{m}\right]_{\mu}$,

$$
\Omega \iota_{*}\left[\iota_{n}, \iota_{m}\right]_{\mu}=\left[\iota_{n}, \iota_{m}\right]
$$

Iteration of the Hilton-Milnor theorem implies that the fibration $\Omega \bar{F}^{n, m} \rightarrow$ $\Omega \bar{E}^{n, m} \stackrel{\Omega \tilde{\pi}}{\longrightarrow} \Omega \Sigma P^{n}\left(p^{r}\right) \times \Omega \Sigma P^{m}\left(p^{r}\right)$ has a section. Hence, there is a unique choice of $\left[\iota_{n}, \iota_{m}\right]_{\mu}$ in $\Omega \bar{F}^{n . m}$ and, if $\Psi=\Omega \tilde{\Psi}$, then

$$
\Psi_{*}\left[\iota_{n}, \iota_{m}\right]_{\mu}=[f, g]_{\mu}
$$

is a well defined definition of the H-based Samelson product in the homotopy of $\Omega F$. Of course, it depends on the multiplication. It is natural with respect to those loop maps of fibration sequences where the map on the base loops is the loop of an H-map.

\section{Exercise}

Show that the Samelson products, the relative products, and the H-based Samelson products are all compatible. That is, verify $9.1 \mathrm{~b}$ ) and 10.1b).

\section{Lie identities for H-based Samelson products}

Here is the detailed verification of the Lie identities for the H-based Samelson products.

Let $p$ be an odd prime and let $F \stackrel{\iota}{\rightarrow} E \stackrel{q}{\rightarrow} B$ be a fibration sequence where $B$ is an H-space with multiplication $\mu: B \times B \rightarrow B$.

Let $\bar{\beta}$ be the composition $P^{n-1}\left(p^{r}\right) \stackrel{q}{\rightarrow} S^{n-1} \stackrel{\iota}{\rightarrow} P^{n}\left(p^{r}\right)$ and let the Bockstein $\beta: \pi_{n}\left(X ; Z / p^{r}\right) \rightarrow \pi_{n-1}(X) \rightarrow \pi_{n-1}\left(X ; Z / p^{r} Z\right)$ be the dual. 
Theorem 13.1 If $w: P^{n}\left(p^{r}\right) \rightarrow \Omega F, x: P^{n}\left(p^{r}\right) \rightarrow \Omega E, \quad y, y_{1}: P^{m}\left(p^{r}\right) \rightarrow$ $\Omega E, \quad z: P^{q}\left(p^{r}\right) \rightarrow \Omega E$ are maps, then

a) $\left[\iota_{*} w, y\right]_{\mu}=[w, y]_{r}$

b) $\left[x, y+y_{1}\right]_{\mu}=[x, y]_{\mu}+\left[x, y_{1}\right]_{\mu}$

c) $[x, y]_{\mu}=-(-1)^{n m}[y, x]_{\mu \cdot T}$

d) if $p>3$, then $\left[x,[y, z]_{\mu}\right]_{r}=\left[[x, y]_{\mu}, z\right]_{r}+(-1)^{n m}\left[y,[x, z]_{\mu}\right]_{r}$

e) $\beta[x, y]_{\mu}=[\beta x, y]_{\mu}+(-1)^{n}[x, \beta y]_{\mu}$.

We shall see that the truth of the above formulas for the usual mod $p^{r}$ Samelson product forces the truth for the H-based products.

a) is proved with the loop of the diagram

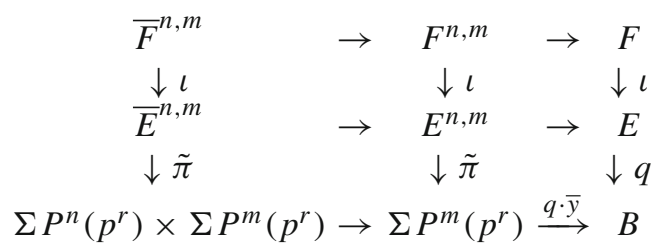

The Samelson products in the fibres map as $\left[\iota_{n}, \iota_{m}\right]_{\mu} \mapsto\left[\iota_{n}, \iota_{m}\right]_{r} \mapsto[w, y]_{r}=$ $\left[\iota_{*} w, y\right]_{\mu}$.

c) is proved with the loop of the diagram

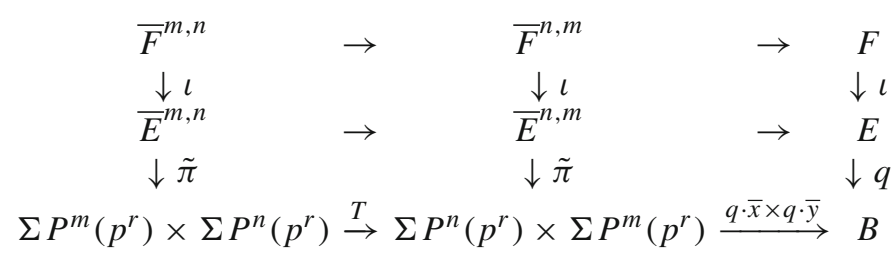

In $\Omega \bar{F}^{n, m}$ we have the equation $\left[\iota_{n}, \iota_{m}\right]=-(-1)^{n m}\left[\iota_{m}, \iota_{n}\right]$. The left hand side maps as $\left[\iota_{n}, \iota_{m}\right] \mapsto[x, y]_{\mu}$ and the right hand side maps as $-(-1)^{n m}\left[\iota_{m}, \iota_{n}\right] \mapsto$ $-(-1)^{n m}\left[\iota_{m}, \iota_{n}\right] \mapsto-(-1)^{n m}[y, x]_{\mu \cdot T}$.

b) is proved with the loops of three diagrams. Consider the map $\Sigma P^{n}\left(p^{r}\right) \times$ $\Sigma P^{m}\left(p^{r}\right) \times \Sigma P^{m}\left(p^{r}\right) \stackrel{q \cdot x \times q \cdot y \times q \cdot y_{1}}{\longrightarrow} B \times B \times B \stackrel{\mu \cdot(1 \times \mu)}{\bar{E}^{n, m, m}} B$ and use it to form the right hand part of the diagram below. The map $\bar{E}^{n, m, m} \rightarrow E$ is the extension to a homotopy equivalent fibre space of the map $\Sigma P^{n}\left(p^{r}\right) \vee \Sigma P^{m}\left(p^{r}\right) \vee$ $\Sigma P^{m}\left(p^{r}\right) \stackrel{\bar{x} \vee \bar{y} \vee \overline{y_{1}}}{\longrightarrow} E$.

The left hand part represents three diagrams depending on whether the map $P^{n}\left(p^{r}\right) \vee P^{m}\left(p^{r}\right) \rightarrow P^{n}\left(p^{r}\right) \vee P^{m}\left(p^{r}\right) \vee P^{m}\left(p^{r}\right)$ is $1 \vee \Delta, 1 \vee 1 \vee *$, or $1 \vee * \vee 1$ 
and whether the map $\Sigma P^{n}\left(p^{r}\right) \times \Sigma P^{m}\left(p^{r}\right) \rightarrow \Sigma P^{n}\left(p^{r}\right) \times \Sigma P^{m}\left(p^{r}\right) \times \Sigma P^{m}\left(p^{r}\right)$ is $(a, b) \mapsto(a, b, b),(a, b) \mapsto(a, b, *)$, or $(a, b) \mapsto(a, *, b)$.

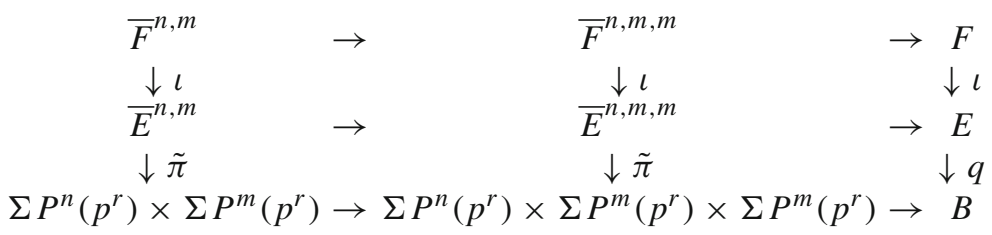

In the homotopy of $\Omega \bar{F}^{n, m, m}$ we have the equation $\left[\iota_{n}, \iota_{m}+\iota_{m}^{\prime}\right]=\left[\iota_{n}, \iota_{m}\right]+\left[\iota_{n}, \iota_{m}^{\prime}\right]$. If we choose the first left hand part, then $\left[\iota_{n}, \iota_{m}\right] \mapsto\left[\iota_{n}, \iota_{m}+\iota_{m}^{\prime}\right] \mapsto\left[x, y+y_{1}\right] \cdot \mu$. The second choice yields $\left[\iota_{n}, \iota_{m}\right] \mapsto\left[\iota_{n}, \iota_{m}\right] \mapsto[x, y]_{\mu}$ and the third choice yields $\left[\iota_{n}, \iota_{m}\right] \mapsto\left[\iota_{n}, \iota_{m}^{\prime}\right] \mapsto\left[x, y_{1}\right]_{\mu}$.

d) is proved by three diagrams. The first one is the loop of

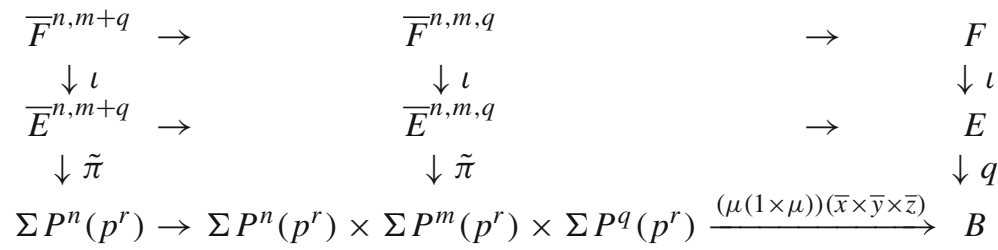

In the second, the right hand column is replaced by the fibration sequence $\bar{F}^{n+m, q} \rightarrow \bar{E}^{n+m, q} \stackrel{\tilde{\pi}}{\rightarrow} \Sigma P^{q}\left(p^{r}\right)$ and, in the third, by the fibration sequence $\bar{F}^{m, n+q} \rightarrow \bar{E}^{m \cdot n+q} \stackrel{\tilde{\pi}}{\rightarrow} \Sigma P^{m}\left(p^{r}\right)$.

In the homotopy of $\Omega \bar{F}^{n, m, q}$ we have the equation

$$
\left[\iota_{n},\left[\iota_{m}, \iota_{q}\right]\right]=\left[\left[\iota_{n}, \iota_{m}\right], \iota_{q}\right]+(-1)^{n m}\left[\iota_{n},\left[\iota_{n}, \iota_{q}\right]\right]
$$

The first diagram yields $\left[\iota_{n}, \iota_{m+q}\right] \mapsto\left[\iota_{n},\left[\iota_{m}, \iota_{q}\right]\right] \mapsto\left[x,[y, z]_{\mu}\right]_{r}$. The second yields $\left.\left[\iota_{n+m}, \iota_{q}\right] \mapsto\left[\left[\iota_{n}, \iota_{m}\right], \iota_{q}\right] \mapsto\left[[x, y]_{\mu}\right]_{r}, z\right]_{r}$ and the third yields $\left.\left[\iota_{m}, \iota_{n+q}\right] \mapsto\left[\iota_{m},\left[\iota_{n}\right], \iota_{q}\right]\right] \mapsto\left[y,[x, z]_{\mu}\right]_{r}$.

e) is proved by the loops of two diagrams

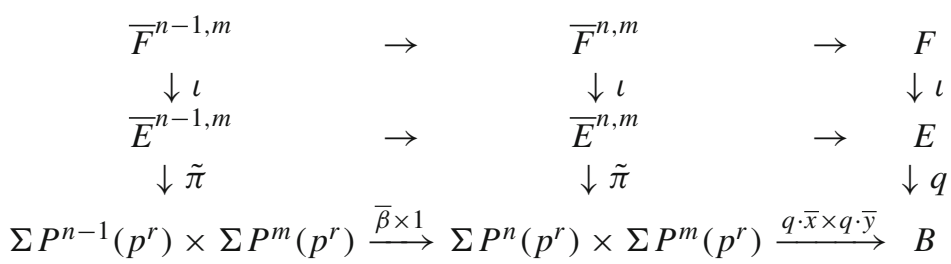




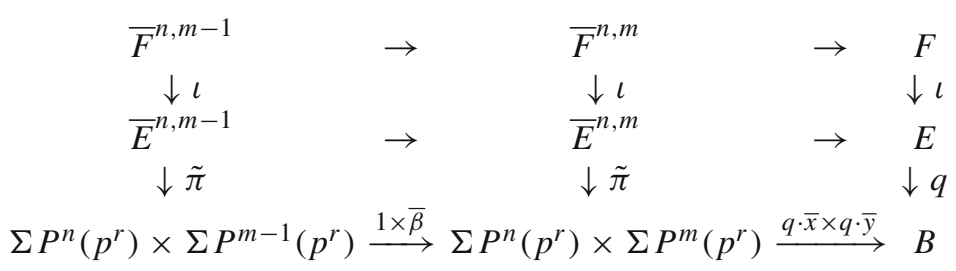

In the homotopy of $\Omega \bar{F}^{n, m}$, we have the equation $\beta\left[\iota_{n}, \iota_{m}\right]=\left[\beta \iota_{n}, \iota_{m}\right]+$ $(-1)^{n}\left[\iota_{n}, \beta \iota_{m}\right]$. The naturality of the Bockstein yields $\beta\left[\iota_{n}, \iota_{m}\right] \mapsto \beta[x, y]_{\mu}$. The first diagram yields $\left[\iota_{n-1}, \iota_{m}\right] \mapsto\left[\beta \iota_{n}, \iota_{m}\right] \mapsto[\beta x, y]_{\mu}$ and the second yields $\left[\iota_{n}, \iota_{m-1}\right] \mapsto\left[\iota_{n}, \beta \iota_{m}\right] \mapsto[x, \beta y]_{\mu}$.

This completes the verification of the Lie identities and the derivation formula for the Bockstein.

\section{Higher order torsion in the homotopy groups of a Moore space}

In this section we show

Theorem 14.1 Let $n \geq 1$. If $p>3$ or if $p=3, r \geq 3$, then there exists a $Z / p^{r+1} Z$ summand in the integral homotopy groups $\pi_{2 p^{k} n-1}\left(P^{2 n+1}\left(p^{r}\right)\right), k \geq 1$.

Proof We convert into a fibration the map $q: P^{2 n+1}\left(p^{r}\right)=S^{2 n-2} \cup_{p^{r}} e^{2 n+1} \rightarrow S^{2 n+1}$ which pinches the bottom cell to a point and consider the resulting fibration sequence up to homotopy

$$
F^{2 n+1}\left\{p^{r}\right\} \stackrel{\iota}{\rightarrow} P^{2 n+1}\left(p^{r}\right) \stackrel{q}{\rightarrow} S^{2 n+1}
$$

In $\pi_{*}\left(\Omega P^{2 n+1}\left(p^{r}\right) ; Z / p Z\right)$ we have a generator $v$ in dimension $2 \mathrm{n}$ and a generator $\mu$ in dimension $2 \mathrm{n}-1$. These two classes have respective $\bmod p$ Hurewicz images $v$ and $u$ in $H_{*}\left(\Omega P^{2 n+1}\left(p^{r}\right) ; Z / p Z\right)$. In the Bockstein spectral sequences we have $\beta^{s} v=\beta^{s} \mu=0, \beta^{s} v=\beta^{s} u=0$ for all $s<r$ and

$$
\beta^{r} v=\mu, \quad \beta^{r} v=u .
$$

Recall the standard alternate notation for a bracket in any graded Lie algebra, $\operatorname{ad}(x)(y)=[x, y]$.

Theorem 14.2 ([5,19]) Suppose the ground ring is a field of characteristic $p>2$. If $x$ is any even degree element of degree $2 n$ in a differential graded Lie algebra, let

$$
\begin{aligned}
\tau_{k}(x) & =\operatorname{ad}(x)^{p^{k}-1}(d x), \\
\sigma_{k}(x) & =\frac{1}{2} \sum_{j=1}^{p^{k}-1} \frac{\left(j, p^{k}-j\right)}{p}\left[\operatorname{ad}(x)^{j-1}(d x), \operatorname{ad}(x)^{p^{k}-j-1}(d x)\right] .
\end{aligned}
$$


These elements have respective degrees of $2 p^{k} n-1$ and $2 p^{k} n-2$. In the universal enveloping algebra,

$$
d\left(x^{p^{k}}\right)=\tau_{k}(x), \quad d\left(\tau_{k}(x)\right)=0, \quad d\left(\sigma_{k}(x)\right)=0 .
$$

Remark 14.3 In the differential graded Lie algebra over $Z_{(p)}, d\left(\tau_{k}(x)\right)$ is divisible by $p$ and $\sigma_{k}(x)=\frac{1}{p} d\left(\tau_{k}(x)\right)$. And, since the above formulas are universally true, they are consequences of the differential Lie identities and hence valid for the relative and $\mathrm{H}$-based Samelson products (if $p>3$ or $p=3, r \geq 3$ and if the base multiplication is homotopy commutative).

The formulas in the above theorem are proved in general by induction. That is left as an exercise. We verify them here only in the simplest case, $p=3, k=1$. Then

$$
\begin{aligned}
d\left(x^{3}\right) & =(d x) x^{2}+x(d x) x+x^{2}(d x), \\
\tau_{1}(x) & =[x,[x, d x]]=x[x, d x]-[x, d x] x \\
& =x(x(d x)-(d x) x)-(x(d x)-(d x) x) x \\
& =x^{2}(d x)-2 x(d x) x+(d x) x^{2} \\
& =x^{2}(d x)+x(d x) x+(d x) x^{2}, \\
d\left(\tau_{1}(x)\right) & =d([x,[x, d x]]) \\
& =[d x,[x, d x]]+[x, d[x, d x]] \\
& =[d x,[x, d x]]+[x,[d x, d x]] \\
& =[d x,[x, d x]]+[[x, d x], d x]+[d x,[x, d x]] \\
& =3[d x,[x, d x]] \\
& =3 \sigma_{1}(x), \\
d\left(\sigma_{1}(x)\right) & =d([d x,[x, d x]]) \\
& =-[d x,[d x, d x]] \\
& =0 .
\end{aligned}
$$

If we return to the general case of $k \geq 1$, but with $p>3$ or $p=3, r \geq 3$, we have in the $r$-th term of the $\bmod p$ Bockstein spectral sequences of $\Omega P^{2 n+1}\left(p^{r}\right)$

a) in homotopy the $\beta^{r}$ cycles $\tau_{k}(v)$ and $\sigma_{k}(v)$.

b) in homology their mod $p$ Hurewicz images $\beta^{r}$ cycles $\tau_{k}(v)$ and $\sigma_{k}(v)$.

c) in homology we have the equation $\beta^{r} v^{p^{k}}=\tau_{k}(v)$. But one of the forms of mod $p$ Hopf invariant one $[10,19,24]$ says that $v^{p^{k}}$ cannot be in the mod p Hurewicz image unless $k=1$. It follows that, unless $k=1$, the element $\sigma_{k}(v)$ cannot be killed by $\beta^{r}$ and hence represents a nontrivial class in the $r+1-s t$ term of the homotopy Bockstein spectral sequence $E^{r+1}$. Hence we have shown that $\sigma_{k}(v)$ represents torsion in $\pi_{*}\left(\Omega P^{2 n+1}\left(p^{r}\right)\right)$ of order $\geq p^{r+1}$ in either dimension $2 p^{k} n-2$ or $2 p^{k} n-3$.

Since $H\left(\Omega P^{2 n+1} ; Z / p Z\right)=E^{r}=T(u, v)$ is an acyclic tensor algebra, it follows that $E^{r+1}=H\left(T(u, v), \beta^{r}\right)=Z / p Z$ and we can see nothing further in this homology 
Bockstein spectral sequence. We look to the homology Bockstein spectral sequence of $\Omega F^{2 n+1}\left\{p^{r}\right\}$ for further information. The relative Samelson products enable us to regard $\tau_{k}(v)$ and $\sigma_{k}(v)$ as cycles in the homotopy Bockstein spectral sequence of $\Omega F^{2 n+1}\left\{p^{r}\right\}$. The absence of powers in the homology of this space makes the representation by the mod $p$ Hurewicz map very strong.

We will prove the next two theorems in later sections.

Theorem 14.4 The mod p homology of the fibration sequence

$$
\Omega F^{2 n+1}\left\{p^{r}\right\} \stackrel{\Omega \iota}{\longrightarrow} \Omega P^{2 n+1}\left(p^{r}\right) \stackrel{\Omega q}{\longrightarrow} \Omega S^{2 n+1}
$$

is the sequence

$$
T\left(a d(v)^{k-1}(u)\right)_{k \geq 1} \stackrel{\subseteq}{\rightrightarrows} T(u, v) \stackrel{v \mapsto \iota, u \mapsto 0}{\longrightarrow} T(\iota) .
$$

The next theorem on the homology Bockstein spectral sequence of the loop space is the mainsail of the work on both of higher torsion in Moore spaces and the exponents of odd dimensional spheres.

Theorem 14.5 The mod p homology Bockstein spectral sequence of $\Omega F^{2 n+1}\left\{p^{r}\right\}$ is

a) $E^{1}=\cdots=E^{r}=T\left(\operatorname{ad}(v)^{k-1}(u)\right)_{k \geq 1}$,

b) $E^{r+1}=S\left(u, \sigma_{k}(v), \tau_{k}(v)\right)_{k \geq 1}=E\left(u, \tau_{k}(v)\right)_{k \geq 1} \otimes P\left(\sigma_{k}(v)\right)_{k \geq 1}=$ the tensor product of an exterior algebra on elements of odd degree and a polynomial algebra on elements of even degree.

c) Up to a unit multiple, $\beta^{r+1} \tau_{k}(v)=\sigma_{k}(v), \quad \beta^{r+1} u=0$ and hence, $Z / p Z=$ $E^{r+2}=\cdots=E^{\infty}$.

It follows that in the homotopy Bockstein spectral sequence of $\Omega F^{2 n+1}\left\{p^{r}\right\}$, we have $\beta^{r+1} \tau_{k}(v) \equiv \sigma_{k}(v)$ modulo the kernel of the Hurewicz map. Consider the factorization of the Hurewicz map

$$
\pi_{*}\left(\Omega P^{2 n+1}\left(p^{r}\right) ; Z / p Z\right)=E^{r} \rightarrow P \subseteq H_{*}\left(\Omega P^{2 n+1}\left(p^{r}\right) ; Z / p Z\right)=E^{r}
$$

where $P$ is the submodule of primitives. Recall that $P$ is generated by the Lie algebra generated by $u$ and $v$ together with the $p$ primary powers of the even degree Lie elements. Hence, $\sigma_{k}(v)$ is not a $\beta^{r+1}$ boundary in $P$.

Therefore, the image of $\beta^{r+1} \tau_{k}(v)$ is nonzero under the map

$$
E^{r+1} \rightarrow H P
$$

The nontriviality of $\beta^{r+1}$ in the homotopy Bockstein spectral sequence of $\Omega P^{2 n+1}\left(p^{r}\right)$ implies the existence of a $Z / p^{r+1} Z$ summand in $\pi_{2 p^{k} n-2}\left(\Omega P^{2 n+1}\left(p^{r}\right)\right)=\pi_{2 p^{k} n-1}$ $\left(P^{2 n+1}\left(p^{r}\right)\right)$.

Remark 14.6 In fact, if $p$ is an odd prime and $n \geq 3$, then $\pi_{*}\left(P^{n}\left(p^{r}\right)\right)$ is annihilated by multiplication by $p^{r+1}[18]$. And Theorem 14.1 is true for all odd primes $p$ and all $r \geq 1[17]$. 


\section{Twisted tensor products and the fibre of the pinch map}

Let $p$ be a prime. The next theorem is perhaps the most surprising in this work on exponents.

Theorem 15.1 a) With $Z$ coefficients $H\left(F^{2 n+1}\left\{p^{r}\right\}\right)$ is torsion free with a basis $\left\{1, g_{1}, \ldots, g_{k}, \ldots\right\}$ where $g_{k}$ has degree $2 n k$. The coproduct is given by

$$
\Delta\left(g_{k}\right)=g_{k} \otimes 1+1 \otimes g_{k}+\sum_{j=1}^{k-1} p^{r}(j, k-j) g_{i} \otimes g_{k-j}
$$

and the action of $H\left(\Omega S^{2 n+1}\right)=T(\iota)$ is given by

$$
\iota^{j} g_{k}=g_{k+j} .
$$

b) The map $\partial: \Omega S^{2 n+1} \rightarrow F^{2 n+1}\left\{p^{r}\right\}$ induces $\partial_{*} \iota^{j}=p^{r} g_{k}$ in homology.

Proof Localize at $q$ where $q$ is a prime different from $p$. Then $P^{2 n+1}\left(p^{r}\right) \simeq *$ and $F^{2 n+1}\left\{p^{r}\right\} \simeq \Omega S^{2 n+1}$. Hence, the integral homology of $F^{2 n+1}\left\{p^{r}\right\}$ has no $q$ torsion.

Cease the localization and consider the $\bmod p$ homology Serre spectral sequence of

$$
\Omega S^{2 n+1} \stackrel{\stackrel{\partial}{\rightarrow}}{\rightarrow} F^{2 n+1}\left\{p^{r}\right\} \stackrel{\iota}{\rightarrow} P^{2 n+1}\left(p^{r}\right)
$$

The action

$$
\mu: \Omega S^{2 n+1} \times F^{2 n+1}\left\{p^{r}\right\} \rightarrow F^{2 n+1}\left\{p^{r}\right\}
$$

makes this spectral sequence into a right module over $H\left(\Omega S^{2 n+1}\right)=T\left(\iota_{2 n}\right)$. We have

$$
E^{2}=H\left(\Omega S^{2 n+1}\right) \otimes H\left(P^{2 n+1}\left(p^{r}\right)\right)=T\left(\iota_{2 n}\right) \otimes\left\langle 1, u_{2 n}, v_{2 n+1}\right\rangle
$$

with differentials $d^{2 n+1}(1 \otimes v)=\iota \otimes 1, \quad d^{2 n+1}(1 \otimes u)=0$. Thus

$$
d^{2 n+1}\left(\iota^{k} \otimes v\right)=\iota^{k+1} \otimes 1, \quad d^{2 n+1}\left(\iota^{k} \otimes u\right)=0
$$

and $H\left(F^{2 n+1}\left\{p^{r}\right\}\right)$ has a basis $1 \otimes 1, \iota \otimes u, \iota^{2} \otimes u, \ldots$ Hence, the integral homology of $F^{2 n+1}\left\{p^{r}\right\}$ has no $p$ torsion, hence, no torsion at all. It is free over $Z$.

Now consider the integral homology Serre spectral sequence of

$$
\Omega S^{2 n+1} \stackrel{\partial}{\rightarrow} F^{2 n+1}\left\{p^{r}\right\} \stackrel{\iota}{\rightarrow} P^{2 n+1}\left(p^{r}\right)
$$

We have

$$
E^{2}=H\left(\Omega S^{2 n+1}\right) \otimes H\left(P^{2 n+1}\left(p^{r}\right)\right)=T\left(\iota_{2 n}\right) \otimes\left\langle 1, u_{2 n}\right\rangle
$$


where $u_{2 n}$ has order $p^{r}$. Hence, $E^{2}=E^{\infty}$ and

$$
\partial_{*}: \bar{H}_{*}\left(\Omega S^{2 n+1}\right) \rightarrow \bar{H}_{*}\left(F^{2 n+1}\left\{p^{r}\right\}\right)
$$

is an injection with cokernel of order $p^{r}$ in each nonzero dimension. If $g_{1}, g_{2}, \ldots$ is a basis for $\bar{H}\left(F^{2 n+1}\left\{p^{r}\right\}\right)$ then $\partial_{*} \iota^{k}=p^{r} g_{k}$ and, since $\partial$ is equivariant, the action is $\iota^{j} g_{k}=g_{k+j}$.

Via the above embedding, we can identify $g_{k}=\frac{1}{p^{r}} \iota^{k}$.

Finally, the coproduct is

$$
\begin{aligned}
\Delta\left(g_{k}\right) & =\Delta\left(\frac{1}{p^{r}} \iota^{k}\right) \\
& =\frac{1}{p^{r}} \Delta\left(\iota^{k}\right)=\frac{1}{p^{r}}(\iota \otimes 1+1 \otimes \iota)^{k} \\
& =g_{k} \otimes 1+1 \otimes g_{k}+\sum_{j=1}^{k-1} p^{r}(j, k-j) g_{i} \otimes g_{k-j} .
\end{aligned}
$$

Let $C$ be the coalgebra with free $Z$ basis $1, u, v$ where $u$ and $v$ are primitive of respective degrees $2 n$ and $2 n+1$ with differential $d v=u, d u=0, d 1=0$. Define a differential $d$ on the twisted tensor product $T(\iota) \otimes_{\tau} C$ by

$$
d\left(\iota^{k} \otimes 1\right)=0, \quad d\left(\iota^{k} \otimes u\right)=0, \quad d\left(\iota^{k} \otimes v\right)=\iota^{k+1} \otimes 1-\iota^{k} \otimes p^{r} u .
$$

Thus, $d$ is equivariant with respect to the left action of $T(\iota)$ and is defined on generators by

$$
d(1 \otimes 1)=0, \quad d(1 \otimes u)=0, \quad d(1 \otimes v)=\iota \otimes 1-1 \otimes p^{r} u .
$$

Theorem 15.2 With $Z$ coefficients there are homology isomorphisms of differential coalgebras

$$
\begin{gathered}
H\left(\Omega S^{2 n+1}\right) \stackrel{\Xi}{\rightarrow} C\left(\Omega S^{2 n+1}\right) \\
H\left(F^{2 n+1}\left\{p^{r}\right\}\right) \stackrel{\Psi}{\leftarrow} T(\iota) \otimes_{\tau} C \stackrel{\Phi}{\rightarrow} C\left(F^{2 n+1}\left\{p^{r}\right\}\right) .
\end{gathered}
$$

Proof Let $x$ be a primitive cycle which represents the generator $\iota$ of $H\left(\Omega S^{2 n+1}\right)$. Define $\Xi: T(\iota)=H\left(\Omega S^{2 n+1}\right) \rightarrow C\left(\Omega S^{2 n+1}\right)$ by $\Xi\left(\iota^{k}\right)=x^{k}$ using the fact that $C\left(\Omega S^{2 n+1}\right)$ is a differential Hopf algebra. Then $\Xi$ is a homology equivalence of differential coalgebras. 
Define a map of coalgebras $\psi: C \rightarrow H\left(F^{2 n+1}\left\{p^{r}\right\}\right)$ by $\psi u=g_{1}, \psi v=0$, $\psi 1=1$. Then $\Psi$ is the composition of coalgebra maps

$$
T(\iota) \otimes C \stackrel{1 \otimes \psi}{\longrightarrow} H\left(\Omega S^{2 n+1}\right) \otimes H\left(F^{2 n+1}\left\{p^{r}\right\}\right) \stackrel{\mu}{\rightarrow} H\left(F^{2 n+1}\left\{p^{r}\right\}\right) .
$$

Since $\Psi$ is equivariant and $\Psi \cdot d=d \cdot \Psi$ on module generators, it is a map of differential coalgebras.

Let $\star: C\left(\Omega S^{2 n+1}\right) \otimes C\left(F^{2 n+1}\left\{p^{r}\right\}\right) \rightarrow C\left(F^{2 n+1}\left\{p^{r}\right\}\right)$ denote the action on chains. Define a map of coalgebras $\phi: C \rightarrow C\left(F^{2 n+1}\left\{p^{r}\right\}\right)$ by $\phi u=c, \phi v=z, \phi 1=1$ where $c$ is a primitive cycle representing $g_{1}, z$ is a primitive chain with $d z=x \star 1-p^{r} c$. Then $\Phi$ is the composition of coalgebra maps

$$
T(\iota) \otimes C \stackrel{\Xi \otimes \phi}{\longrightarrow} C\left(\Omega S^{2 n+1}\right) \otimes C\left(F^{2 n+1}\left\{p^{r}\right\}\right) \stackrel{\mu}{\rightarrow} C\left(F^{2 n+1}\left\{p^{r}\right\}\right) .
$$

Since $\Psi$ is equivariant and $\Psi \cdot d=d \cdot \Psi$ on module generators, it is a map of differential coalgebras. It is clearly a homology isomorphism.

\section{Lie algebras and the loops on the fibre of the pinch map}

Let $0 \rightarrow K \rightarrow L \stackrel{q}{\rightarrow} M \rightarrow 0$ be a short exact sequence of graded Lie algebras, free as modules over a commutative ring $R$ in which 2 is a unit. Assume the ring has the property that projective modules are free.

The next four theorems enable the computation of the homology of the fibres in certain fibration sequences which are totally non homologous to zero. Proofs can be found in [19].

Theorem 16.1 $U L \simeq U K \otimes U M$ as a $U K$ module and $U M$ comodule. (See section 8.3 in [19].)

Theorem 16.2 Let $\Omega F \stackrel{\Omega \iota}{\longrightarrow} \Omega E \stackrel{\Omega q}{\longrightarrow} \Omega B$ be a fibration sequence of loop maps and suppose that $\Omega q$ induces the homologymap $U L \rightarrow U M$. Then there is an isomorphism $U K \rightarrow H(\Omega F)$. (See section 10.24 in [19].)

These theorems imply that

$\operatorname{Cotor}^{U M}(U L, R)=\operatorname{Cotor}_{0}^{U M}(U L, R)=U L \square_{U M} R=U K \otimes U M \square_{U M} R=U K$

with Cotor concentrated in homological degree 0. And, hence that the EilenbergMoore spectral sequence [6] collapses and the edge homomorphism $H(\Omega F) \stackrel{\simeq}{\rightarrow} U K$ is an isomorphism.

Theorem 16.3 Suppose that $K$ is a subalgebra of a free Lie algebra which is a split summand as a module. Then K is a free Lie algebra. (See section 8.7 in [19].)

Generators of such subalgebras are often determined by 
Theorem 16.4 a) The kernel of the map of free Lie algebras $L\left(x, x_{\alpha}\right) \rightarrow$ $L(x), \quad x_{\alpha} \mapsto 0$ is the free Lie algebra $L\left(\operatorname{ad}(x)^{k}\left(x_{\alpha}\right)\right)_{k \geq 0}$.

b) If $x$ has odd degree, then the kernel of the map to the abelian Lie algebra $L\left(x, x_{\alpha}\right) \rightarrow\langle x\rangle, \quad x_{\alpha} \mapsto 0$ is the free Lie algebra $L\left(x_{\alpha},\left[x, x_{\alpha}\right],[x, x]\right)$.

(See section 8.7 in [19].)

If $p$ is a prime, then with coefficients $Z / p^{s} Z, \quad s \leq r$ the map $\Omega P^{2 n+1}\left(p^{r}\right) \rightarrow$ $\Omega S^{2 n+1}$ induces the map of universal enveloping algebras

$$
U L(u, v)=T(u, v) \rightarrow U L(v)=T(v)
$$

with $\operatorname{deg}(u)=2 n, \operatorname{deg}(v)=2 n+1$. Hence,

Corollary 16.5 Suppose $p$ is an odd prime. With coefficients $Z / p^{s} Z, \quad s \leq r$,

$$
H\left(\Omega F^{2 n+1}\left\{p^{r}\right\}\right)=U L\left(a d(v)^{k-1}(u)\right)_{k \geq 1}=T\left(a d(v)^{k-1}(v)(u)\right)_{k \geq 1} .
$$

In the mod p homology Bockstein spectral sequence, the Bockstein differentials are given by $\beta^{r} v=u, \beta^{s} v=\beta^{s} u=0, s \leq r$.

\section{The $\bmod p$ homology Bockstein spectral sequence of the loops on the fibre of the pinch map}

The computation of the mod $p$ homology of $\Omega^{2} S^{2 n+1}$ is due independently to Toda [26] and Moore [15].

Theorem 17.1 a) With any coefficients $R, H\left(\Omega^{2} S^{2 n+1}\right)=\operatorname{Cotor}^{H\left(\Omega S^{2 n+1}\right)}(R, R)$. b) With $Z / p Z$ coefficients, $H\left(\Omega^{2} S^{2 n+1}\right)=E\left(u, \tau_{1}, \tau_{2}, \ldots\right) \otimes P\left(\sigma_{1}, \sigma_{2}, \ldots\right)$ where $u$ has degree $2 n-1, \tau_{k}$ has degree $2 p^{k} n-1, \sigma_{k}$ has degree $2 p^{k} n-2$, and the Bockstein are $\beta^{1} \tau_{k}=\sigma_{k}, \beta^{s} u=0$ for all $s \geq 1$.

c) The $p$ torsion in $H\left(\Omega^{2} S^{2 n+1} ; Z\right)$ is annihilated by $p$.

Proof Since there is a homology equivalence of differential coalgebras $T\left(\iota_{2 n}\right) \rightarrow$ $C\left(\Omega S^{2 n+1}\right)$,

$$
H\left(\Omega^{2} S^{2 n+1}\right)=\operatorname{Cotor}^{C\left(\Omega S^{2 n+1}\right)}(R, R)=\operatorname{Cotor}^{H\left(\Omega S^{2 n+1}\right)}(R, R) .
$$

With $Z / p Z$ coefficients,

$$
\begin{aligned}
\operatorname{Cotor}^{H\left(\Omega S^{2 n+1}\right)}(Z / p Z, Z / p Z) & =\operatorname{Cotor}^{T(\iota)}(Z / p Z, Z / p Z) \\
& =E\left(u, \tau_{1}, \tau_{2}, \ldots\right) \otimes P\left(\sigma_{1}, \sigma_{2}, \ldots\right) .
\end{aligned}
$$

In this computation the elements $\tau_{k}$ are identified as the transgressions of the powers $\iota^{p^{k}}$. The result on $p$ torsion is an immediate consequence of the collapse of the homology Bockstein spectral sequence, $E^{2}=\cdots=E^{\infty}=E(u)$. 
The above result on the $p$ torsion in the homology of $\Omega^{2} S^{2 n+1}$ is applied in the following result to bound the order of the $p$ torsion in the homology of $\Omega F^{2 n+1}\left\{p^{r}\right\}$.

Theorem 17.2 a) With any coefficients $R, H\left(\Omega F^{2 n+1}\left\{p^{r}\right\}\right)=\operatorname{Cotor}^{H\left(F^{2 n+1}\left\{p^{r}\right\}\right)}$ $(R, R)$.

b) The $p$ torsion in $H\left(\Omega F^{2 n+1}\left\{p^{r}\right\}\right)$ is annihilated by $p^{r+1}$.

Proof The homology equivalences of differential coalgebras

$$
H=H\left(F^{2 n+1}\left\{p^{r}\right\}\right) \stackrel{\Psi}{\leftarrow} T(\iota) \otimes_{\tau} C \stackrel{\Phi}{\rightarrow} C\left(F^{2 n+1}\left\{p^{r}\right\}\right)
$$

show that

$$
H\left(\Omega F^{2 n+1}\left\{p^{r}\right\}\right)=\operatorname{Cotor}^{C\left(F^{2 n+1}\left\{p^{r}\right\}\right)}(R, R)=\operatorname{Cotor}^{H}(R, R) .
$$

Let $T=H\left(\Omega S^{2 n+1} ; Z\right)$. We have shown that there are subcoalgebras $T \subset H \subset$ $T \otimes Q$ with augmentation ideals related by $\bar{H}=\frac{1}{p^{r}} \bar{T}$.

Consider the cobar construction

$$
\Omega(H)=\oplus_{k \geq 0} \Omega_{k}(H)
$$

with $\Omega_{k}(H)=\otimes \bar{H}^{k}$ and differential $d: \Omega_{k}(H) \rightarrow \Omega_{k+1}(H)$. Let $Z_{k}(H) \subseteq \Omega_{k}(H)$ be the cycles of tensor length $k$ and let $B_{k}(H) \subseteq \Omega_{k}(H)$ be the boundaries of length $k$. Then

$$
\begin{gathered}
\Omega_{k}(H)=\frac{1}{p^{r k}} \Omega_{k}(T), \quad Z_{k}(H)=\frac{1}{p^{r k}} Z_{k}(T), \quad B_{k}(H)=\frac{1}{p^{r(k-1)}} Z_{k}(T), \\
Z_{k}(H) / B_{k}(H)=\frac{1}{p^{r k}} Z_{k}(T) / \frac{1}{p^{r(k-1)}} Z_{k}(T) \simeq Z_{k}(T) / p^{r} B_{k}(T) .
\end{gathered}
$$

Since $Z_{k}(T) / B_{k}(T) \subseteq H\left(\Omega^{2} S^{2 n+1}\right)$ has $p$ torsion annihilated by $p$ and $B_{k}(T) / p^{r}$ $B_{k}(T)$ is torsion annihilated by $p^{r}$, this implies that $\oplus_{k \geq 0} Z_{k}(H) / B_{k}(H)=$ $H\left(\Omega F^{2 n+1}\left\{p^{r}\right\}\right)$ has $p$ torsion annihilated by $p^{r+1}$.

Theorem 17.3 The homology Bockstein spectral sequence of $\Omega F^{2 n+1}\left\{p^{r}\right\}$ is:

$$
\begin{gathered}
E^{1}=E^{2}=\cdots=E^{r}=T\left(\operatorname{ad}(v)^{k}(u)\right)_{k \geq 0}, \quad \operatorname{deg}(v)=2 n, \quad \operatorname{deg}(u)=2 n-1 . \\
E^{r+1}=H\left(T\left(\operatorname{ad}(v)^{k}(u)\right)_{k \geq 0}, \beta^{r}\right)=E\left(u, \tau_{1}(v), \tau_{2}(v), \ldots\right) \otimes P\left(\sigma_{1}(v), \sigma_{2}(v), \ldots\right)
\end{gathered}
$$

where $u$ has degree $2 n-1, \tau_{k}(v)=\tau_{k}=\operatorname{ad}(v)^{p^{k}-1}(v)(u)$ has degree $2 p^{k} n-1$, $\sigma_{k}(v)=\sigma_{k}=\frac{1}{2} \sum_{j=1}^{p^{k}-1} \frac{\left(j, p^{k}-j\right)}{p}\left[\operatorname{ad}(v)^{j-1}(u), \operatorname{ad}(v)^{p^{k}-j-1}(u)\right]$ has degree $2 p^{k} n-$ 2 , and the Bocksteins are, up to a unit multiplier, $\beta^{r+1} \tau_{k}=\sigma_{k}, \beta^{s} u=0$ for all $s \geq 1$.

$$
E^{r+2}=\cdots=E^{\infty}=Z / p Z .
$$


Proof In the sequence $T\left(a d(v)^{k-1}(u)\right) \rightarrow T(u, v) \rightarrow T(\iota), T(u, v)$ is acyclic and is a twisted tensor product $T\left(\operatorname{ad}(v)^{k-1}(u)\right)_{k \geq 1} \otimes_{\tau} T(\iota)$ as a $T\left(\operatorname{ad}(v)^{k-1}(u)\right)_{k \geq 1}$ comodule and a $T(\iota)$ comodule. In fact, the existence of the twisting $\tau$ is a consequence of general principles about short exact sequences of differential graded Lie algebras. That is all we need but by using results in [19] it is possible to be explicit about the differential in $T\left(a d(v)^{k-1}(u)\right)_{k \geq 1} \otimes_{\tau} T(v)$ :

$$
\begin{aligned}
d(y \otimes 1)= & d y \otimes 1, \quad d\left(y \otimes v^{m}\right)=d y \otimes v^{m} \\
& +(-1)^{\operatorname{deg}(y)} y \sum_{j=0}^{m-1}(j, m-j) a d(v)^{m-j-1}(u) \otimes v^{j}
\end{aligned}
$$

for all $y$ in $T\left(\operatorname{ad}(v)^{k-1}(u)\right)_{k \geq 1}$.

Hence, with $Z / p Z$ coefficients,

$$
\begin{aligned}
E^{r+1} & =H\left(T\left(\operatorname{ad}(v)^{k-1}(u)\right)_{k \geq 1}, \beta^{r}\right) \\
& =\operatorname{Cotor}^{T(v)}(Z / p Z, Z / p Z) \\
& =E\left(u, \tau_{1}, \tau_{2}, \ldots\right) \otimes P\left(\sigma_{1}, \sigma_{2}, \ldots\right)
\end{aligned}
$$

where $\tau_{k}$ is a primitive element of degree $2 p^{k} n-1$ and $\sigma_{k}$ is a primitive element of degree $2 p^{k} n-2$.

The generators $\tau_{k}$ are the transgressions of the powers $v^{p^{k}}$. Since $\beta^{r} v^{p^{k}}=\tau_{k}(v)$, that is, $\beta^{r}\left(1 \otimes v^{p^{k}}\right)=a d(v)^{p^{k}-1}(v)(u) \otimes 1$, it follows that $\tau_{k}=\tau_{k}(v)$. Note that the differential $\beta^{r}$ is compatible with the trigrading consisting of total degree, the degree in $v$, and the degree in $u$. Hence, this trigrading passes to the homology $H\left(T\left(a d(v)^{k-1}(u)\right)_{k \geq 1}, \beta^{r}\right)$. Since $\sigma_{k}(v)$ has degree 2 in $u$ it cannot be a boundary. And inspection shows that its homology class cannot be a decomposable element. Hence, up to a unit multiple $\sigma_{k}=\sigma_{k}(v)$.

The previous theorem says that the $p$ torsion in the homology of $\Omega F^{2 n+1}\left\{p^{r}\right\}$ is of order $p^{r+1}$. Hence, the homology Bockstein spectral sequence collapses. There is a homotopy equivalence of rationalizations,

$$
\Omega F^{2 n+1}\left\{p^{r}\right\} \otimes Q \simeq \Omega S^{2 n+1} \otimes Q,
$$

Hence, $E^{r+2}=\cdots=E^{\infty}=Z / p Z$. The only way this can occur is that, up to a unit multiple, $\beta^{r+1} \tau(v)=\sigma_{k}(v)$.

\section{A tensor product splitting of a universal enveloping algebra}

The corollary at the end of this section is the algebraic form of the splitting of the loop space $\Omega F^{2 n+1}\left\{p^{r}\right\}$. In this section the ground ring will be $Z / p Z$.

Theorem 18.1 If $L=L\left(a d(v)^{k-1}(u)\right)_{k \geq 1}$, there is a decreasing sequence of differential subalgebras

$$
L=L_{0} \supset L_{1} \supset L_{2} \supset L_{3} \supset \cdots
$$


with

a) if we write $\tau_{k}(v)=\tau_{k}, \sigma_{k}(v)=\sigma_{k}$, there exist short exact sequences of differential graded Lie algebras

$$
\begin{aligned}
0 & \rightarrow L_{1} \rightarrow L_{0} \rightarrow\langle u\rangle \rightarrow 0 \\
0 \rightarrow L_{k+1} & \rightarrow L_{k} \rightarrow\left\langle\sigma_{k}, \tau_{k}\right\rangle \rightarrow 0, \quad k \geq 1
\end{aligned}
$$

(The $\left\langle\sigma_{k}, \tau_{k}\right\rangle$ are abelian Lie algebras with 0 differential.)

b)

$$
\begin{gathered}
H(U L)=H\left(U L_{0}\right)=E\left(u, \tau_{1}, \tau_{2}, \ldots\right) \otimes P\left(\sigma_{1}, \sigma_{2}, \ldots\right)=S\left(u, \tau_{j}, \sigma_{j}\right)_{j \geq 1} \\
H\left(U L_{k}\right)=E\left(\tau_{k},, \tau_{k+1}, \ldots\right) \otimes P\left(\sigma_{k}, \sigma_{k+1}, \ldots\right)=S\left(\tau_{j}, \sigma_{j}\right)_{j \geq k}, \quad k \geq 1
\end{gathered}
$$

c) if $L_{\infty}=\cap_{k \geq 0} L_{k}$ then $H\left(U L_{\infty}\right)=Z / p Z$ and $L_{\infty}=L\left(x_{\alpha}, \beta^{r} x_{\alpha}\right)$ is a free Lie algebra on an acyclic set of generators.

We will need the following lemma which we assume here without proof [19].

Lemma 18.2 (Algebraic Hurewicz theorem) Let A be a connected supplemented differential algebra with A isomorphic as an algebra to a tensor algebra. Suppose that the homology of the indecomposables is n connected, that is, $H_{k} Q(A)=0$ for $k<n$, then the natural map

$$
\bar{H}_{k} A \rightarrow H_{k} Q(A)
$$

is an isomorphism if $k<2 n$ and an epimorphism if $k=2 n$.

We proceed to prove a) and b) by induction on $k$.

Since $u$ is a generator of $L=L_{0}=L\left(u, \operatorname{ad}(v)(u), \operatorname{ad}(v)^{2}(u), \ldots\right)$, sending all other generators to 0 gives an epimorphism of differential graded Lie algebras $L_{0} \rightarrow\langle u\rangle$. If $L_{1}=$ kernel $L_{0} \rightarrow\langle u\rangle$, then $L_{0}$ is a free Lie algebra and the exact sequence shows that

$$
U L_{0}=U\langle u\rangle \otimes U L_{1}=E(u) \otimes U L_{1}
$$

as $U\langle u\rangle=E(u)$ coalgebras and $U L_{1}$ modules. In fact, there is an isomorphism to a twisted tensor product

$$
U L_{0}=U\langle u\rangle \otimes_{\tau} U L_{1}
$$

Hence,

$$
\begin{aligned}
H U L_{1} & =\text { Cotor }^{U\langle u\rangle}(Z / p Z, H U L) \\
& =Z / p Z \square_{U\langle u\rangle} H U L \\
& =E\left(\tau_{k}\right)_{k \geq 1} \otimes P\left(\sigma_{k}\right)_{k \geq 1}=S\left(\tau_{j}, \sigma_{j}\right)_{j \geq 1}
\end{aligned}
$$


The inductive hypothesis is that we have constructed differential sub Lie algebras

$$
L_{k} \subset L_{k-1} \subset \cdots \subset L
$$

such that

$$
H U L_{k}=E\left(\tau_{k}, \tau_{k+1}, \ldots\right) \otimes P\left(\sigma_{k}, \sigma_{k+1}, \ldots\right) .
$$

The algebraic Hurewicz theorem implies that $\tau_{k}$ and $\sigma_{k}$ are generators which represent nonzero homology classes. They are not even boundaries modulo indecomposables. Therefore there is an epimorphism of differential Lie algebras

$$
L_{k} \rightarrow\left\langle\sigma_{k}, \tau_{k}\right\rangle
$$

where the abelian Lie algebra $\left\langle\sigma_{k}, \tau_{k}\right\rangle$ has 0 differential.

If $L_{k+1}=$ kernel $L_{k} \rightarrow\left\langle\sigma_{k}, \tau_{k}\right\rangle$, then $L_{k+1}$ is a free Lie algebra,

$$
U L_{k}=U\left\langle\sigma_{k}, \tau_{k}\right\rangle \otimes U L_{k+1}
$$

and, since

$$
H U L_{k}=S\left(\sigma_{k}, \tau_{k}\right) \otimes S\left(\sigma_{j}, \tau_{j}\right)_{j \geq k+1}
$$

as $S\left(\sigma_{k}, \tau_{k}\right)$ comodules,

$$
\begin{aligned}
H U L_{k+1} & =\text { Cotor }^{U\left\langle\sigma_{k}, \tau_{k}\right\rangle}\left(Z / p Z, H U L_{k}\right) \\
& =Z / p Z \square_{U\left\langle\sigma_{k}, \tau_{k}\right\rangle} H U L_{k} \\
& =E\left(\tau_{j}\right)_{j \geq k+1} \otimes P\left(\sigma_{k}\right)_{j \geq k+1} .
\end{aligned}
$$

The filtration $\cdots \subseteq L_{k} \subset L_{k-1} \subseteq \cdots \subseteq L$ is finite in each degree. Hence, if $L_{\infty}=\cap_{k \geq 0} L_{k}$, then $L_{\infty}$ is a free Lie algebra with

$$
H U L_{\infty}=\bigcap_{k \geq 0} U L_{k}=Z / p Z .
$$

The algebraic Hurewicz theorem shows $H Q L_{\infty}=H Q U L_{\infty}=0$. It follows that $L_{\infty}$ is generated by an acyclic set of generators, that is, $L=L\left(x_{\alpha}, \beta^{r} x_{\alpha}\right)_{\alpha}$.

Corollary 18.3 As a differential coalgebra

$$
\begin{aligned}
U L & =E(u) \otimes \bigotimes_{k \geq 1} S\left(\sigma_{k}, \tau_{k}\right) \otimes U L_{\infty} \\
& =E\left(u, \tau_{k}\right)_{k \geq 1} \otimes P\left(\sigma_{k}\right)_{k \geq 1} \otimes T\left(x_{\alpha}, \beta^{r} x_{\alpha}\right)_{\alpha} .
\end{aligned}
$$




\section{Product splitting of the loops on the fibre of the pinch map}

We realize the previous tensor decomposition of homology by a geometric splitting of a loop space into an infinite product. Easier cases are presented in the exericises at the end of this section.

Let $S^{2 n+1}\left\{p^{r}\right\} \rightarrow S^{2 n+1} \stackrel{p^{r}}{\rightarrow} S^{2 n+1}$ be a fibration sequence up to homotopy. The mod $p$ homology Serre spectral sequence of the fibration sequence $\Omega S^{2 n+1} \rightarrow$ $S^{2 n+1}\left\{p^{r}\right\} \rightarrow S^{2 n+1}$ is a spectral sequence of left $H\left(\Omega S^{2 n+1}\right)=T(\iota)$ modules. It collapses and hence

Lemma 19.1 With $Z / p Z$ coefficients

$$
H\left(S^{2 n+1}\left\{p^{r}\right\}\right)=S(u, v), \quad \operatorname{deg}(u)=2 n, \quad \operatorname{deg}(v)=2 n+1
$$

as a coalgebra and left $S(u)=P(u)=T(u)$ module. The generators are primitive and $\beta^{r} v=u$.

Consider the map of fibration sequences

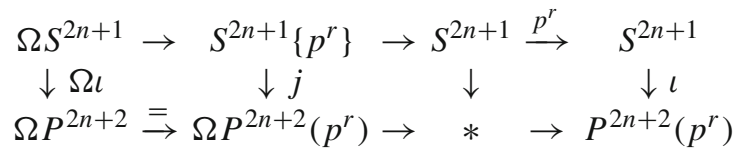

It follows that the map $S^{2 n+1}\left\{p^{r}\right\} \stackrel{j}{\rightarrow} \Omega P^{2 n+1}\left(p^{r}\right)$ induces the map in mod p homology $u^{k} \mapsto u^{k}, \quad u^{k} v \mapsto u^{k} v$.

In order to avoid problems with the Jacobi identity, we will assume $p>3$.

Corollary 19.2 There is a homotopy equivalence of spaces localized at an odd prime $p>3$

$$
\Psi: S^{2 n-1} \times \prod_{k \geq 1} S^{2 p^{k} n-1}\left\{p^{r+1}\right\} \times \Omega \Sigma \bigvee_{\alpha} P^{n_{\alpha}}\left(p^{r}\right) \stackrel{\simeq}{\rightarrow} \Omega F^{2 n+1}\left\{p^{r}\right\}
$$

where the product is the weak product, that is, the direct limit of all the finite products, and $P^{n_{\alpha}}\left(p^{r}\right)$ is an infinite collection of Moore spaces with only finitely many in each dimension.

Proof Let $\mu: S^{2 n-1} \rightarrow \Omega F^{2 n+1}\left\{p^{r}\right\}$ represent a generator so that the Hurewicz map is $\phi v=u$ is a generator of $Z=H_{2 n-1}\left(\Omega F^{2 n+1}\left\{p^{r}\right\}\right)$ In other words, the $\bmod p$ homology image of $\mu$ is the exterior algebra $E(u) \subset H\left(\Omega F^{2 n+1}\left\{p^{r}\right\}\right)$.

Let $\tau_{k}(v): P^{2 p^{k} n-1}\left(p^{r}\right) \rightarrow \Omega F^{2 n+1}\left\{p^{r}\right\}$ and $\sigma_{k}(\nu): P^{2 p^{k} n-2}\left(p^{r}\right) \rightarrow$ $\Omega F^{2 n+1}\left\{p^{r}\right\}$ be the relative Samelson products. Since the Bockstein

$$
\beta \cdot \tau_{k}(\nu): P^{2 p^{k} n-2}(p) \stackrel{\bar{\beta}}{\rightarrow} P^{2 p^{k} n-1}\left(p^{r}\right) \stackrel{\tau_{k}(\nu)}{\longrightarrow} \Omega F^{2 n+1}\left\{p^{r}\right\}
$$


is null homotopic, there is a factorization

$$
\tau_{k}(v)=\overline{\tau_{k}(v)} \cdot \bar{\rho}: P^{2 p^{k} n-1}\left(p^{r}\right) \stackrel{\bar{\rho}}{\rightarrow} P^{2 p^{k} n-1}\left(p^{r+1}\right) \stackrel{\overline{\tau_{k}(v)}}{\longrightarrow} \Omega F^{2 n+1}\left\{p^{r}\right\}
$$

The mod $p$ Hurewicz image of $\tau_{k}(v)$ is $\tau_{k}(v)$ and the coreduction map $\bar{\rho}$ is degree 1 on the top cell. Therefore, on the $2 p^{k} n-1$ dimensional generator $e$ of the mod $p$ homology of $P^{2 p^{k} n-1}\left(p^{r+1}\right),{\overline{\tau_{k}(v)_{*}}}_{*}(e)=\tau_{k}(v)$ in the $\bmod p$ homology of $\Omega F^{2 n+1}\left\{p^{r}\right\}$. But then, up to a unit multiple, ${\overline{\tau_{k}(v)}}_{*}\left(\beta^{r+1} e\right)=\beta^{r+1} \tau_{k}(v)=\sigma_{k}(v)$ on the $2 p^{k} n-2$ dimensional generator.

Thus, the reduced mod $p$ homology image of $\overline{\tau_{k}}: P^{2 p^{k} n-1}\left(p^{r+1}\right) \rightarrow \Omega F^{2 n+1}\left\{p^{r}\right\}$ is $\left\langle\tau_{k}(v), \sigma_{k}(v)\right\rangle$.

Let $\psi: S^{2 p^{k} n+1}\left\{p^{r+1}\right\} \stackrel{j}{\rightarrow} \Omega \Sigma P^{2 p^{k} n-1}\left(p^{r+1}\right) \rightarrow \Omega F^{2 n+1}\left\{p^{r}\right\}$ be the composition with the multiplicative extension. In $\bmod p$ homology the image of $\psi$ is $\left.S\left(\tau_{k}(v), \sigma_{k}(v)\right)\right)$.

Use the multiplication to form the map

$$
\begin{aligned}
S^{2 n-1} & \times \prod_{k=1}^{\ell} S^{2 p^{k} n-1}\left\{p^{r+1}\right\} \stackrel{\mu \times \prod \psi}{\longrightarrow} \Omega F^{2 n+1}\left\{p^{r}\right\} \\
& \times \prod \Omega F^{2 n+}\left\{p^{r}\right\} \stackrel{\text { mult }}{\longrightarrow} \Omega F^{2 n+1}\left\{p^{r}\right\}
\end{aligned}
$$

Let $\ell$ go to infinity and extend this to the weak product

$$
S^{2 n-1} \times \prod_{k=1}^{\infty} S^{2 p^{k} n-1}\left\{p^{r+1}\right\} \stackrel{\mu \times \prod \psi}{\longrightarrow} \Omega F^{2 n+1}\left\{p^{r}\right\}
$$

so that the mod $p$ homology image is $S\left(u, \tau_{j}(v), \sigma_{j}(v)\right)_{j \geq 1}$.

Represent the Lie brackets $x_{\alpha}$ by relative Samelson products $\chi_{\alpha}: P^{n_{\alpha}}\left(p^{r}\right) \rightarrow$ $\Omega F^{2 n+1}\left\{p^{r}\right\}$. The reduced mod $p$ homology image of $\chi_{\alpha}$ is $\left\langle x_{\alpha}, \beta^{r} x_{\alpha}\right\rangle$.

Form the bouquet of maps $\bigvee_{\alpha} \chi_{\alpha}: \bigvee P^{n_{\alpha}}\left(p^{r}\right) \rightarrow \Omega F^{2 n+1}\left\{p^{r}\right\}$, and let

$$
\Upsilon: \Omega \Sigma \bigvee_{\alpha} P^{n_{\alpha}}\left(p^{r}\right) \rightarrow \Omega F^{2 n+1}\left\{p^{r}\right\}
$$

be the multiplicative extension. The image of $\Upsilon$ in $\bmod p$ homology is $T\left(x_{\alpha}, \beta^{r} x_{\alpha}\right)_{\alpha}$.

Finally multiplying the maps produces an isomorphism of $\bmod p$ homology

$$
S^{2 n-1} \times \prod_{k \geq 1} S^{2 p^{k} n-1}\left\{p^{r+1}\right\} \times \Omega \Sigma \bigvee_{\alpha} P^{n_{\alpha}}\left(p^{r}\right) \rightarrow \Omega F^{2 n+1}\left\{p^{r}\right\}
$$

Hence, this is an equivalence of spaces localized at an odd prime $p>3$. 


\section{Exercises}

(1) Use the short exact sequence of graded Lie algebras

$$
0 \rightarrow L\left(x^{2}\right) \rightarrow L(x) \rightarrow\langle x\rangle \rightarrow 0, \quad \operatorname{deg}(x)=2 n+1
$$

to prove Serre's theorem. Localized away from 2, there is a homotopy equivalence

$$
S^{2 n+1} \times \Omega S^{4 n+3} \stackrel{\simeq}{\rightarrow} \Omega S^{2 n+2} .
$$

(2) Use the short exact sequence of differential graded Lie algebras

$$
0 \rightarrow L\left(a d(d x)^{k}\left(x^{2}\right), a d(d x)^{k}[d x, x]\right)_{k \geq 0} \rightarrow L(x, d x) \rightarrow\langle x, d x\rangle \rightarrow 0
$$

if $\operatorname{deg}(x)=2 n+1$, to prove the product decomposition for the loops on an even dimensional Moore space. If $p$ is an odd prime, then there is a homotopy equivalence

$$
S^{2 n+1}\left\{p^{r}\right\} \times \Omega \vee_{k=0}^{\infty} P^{4 n+2 k n+3}\left(p^{r}\right) \stackrel{\simeq}{\rightarrow} \Omega P^{2 n+2}\left(p^{r}\right) .
$$

Remark 19.3 The above results show that at odd primes the homotopy theory of Moore spaces and of spheres can be reduced to the odd dimensional cases.

\section{Exponents of the homotopy groups of spheres}

A fundamental lemma says that a homotopy commutative diagram may be expanded into a diagram where the rows and columns are fibration sequences up to homotopy $[4,19]$. An example of this is:

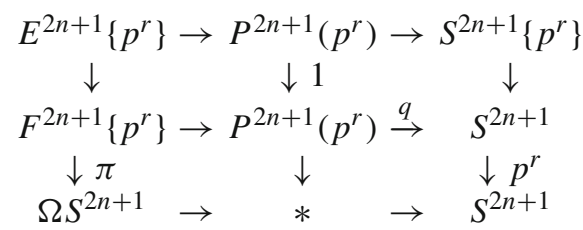

If we loop this and extend the fibrations to the left, we get the diagram of loop maps where all the rows and columns are fibration sequences.

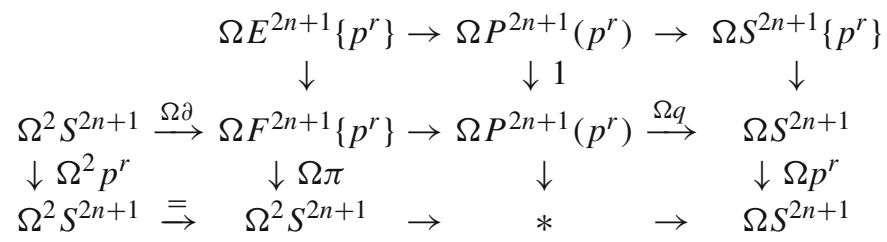


Theorem 20.1 Localized at an odd prime $p$, the map $S^{2 n+1}\left\{p^{r}\right\} \rightarrow S^{2 n+1}$ is an $H$ map of homotopy commutative H spaces [19].

It follows that we can use H-based Samelson products in the top and middle rows. Hence, the relative Samelson products of the previous section $\chi_{\alpha}$, and $\tau(v), \sigma_{k}(v)$ all lift to $\Omega E^{2 n+1}\left\{p^{r}\right\}$ and, if $p>3$, so do the extensions $\overline{\tau_{k}(v)}$. It follows that the maps

$$
\prod_{k \geq 1} S^{2 p^{k} n-1}\left\{p^{r+1}\right\} \times \Omega \Sigma \bigvee_{\alpha} P^{n_{\alpha}}\left(p^{r}\right) \rightarrow \Omega F^{2 n+1}\left\{p^{r}\right\}
$$

lift to $\Omega E^{2 n+1}\left\{p^{r}\right\}$. Hence, the map $\Omega \pi$ factors as

$$
\begin{aligned}
\Omega \pi=\Sigma^{2} \cdot \bar{\pi}: \Omega F^{2 n+1}\left\{p^{r}\right\} & \simeq S^{2 n-1} \times \prod_{k \geq 1} S^{2 p^{k} n-1}\left\{p^{r+1}\right\} \times \Omega \bigvee_{\alpha} P^{n_{\alpha}}\left(p^{r}\right) \\
& \stackrel{\bar{\pi}}{\rightarrow} S^{2 n_{1}} \stackrel{\Sigma^{2}}{\longrightarrow} \Omega^{2} S^{2 n+1}
\end{aligned}
$$

where we have identified $\Omega F^{2 n+1}\left\{p^{r}\right\}$ with its product decomposition by the homotopy equivalence, $\bar{\pi}$ is the projection on the first factor, and $\Sigma^{2}$ is the double suspension.

Set $\pi_{r}=\bar{\pi} \cdot \Omega \partial: \Omega^{2} S^{2 n+1} \rightarrow \Omega F^{2 n+1}\left\{p^{r}\right\} \rightarrow S^{2 n-1}$. The above diagram yields

Theorem 20.2 If $p>3$, then the map $\pi_{r}: \Omega^{2} S^{2 n+1} \rightarrow S^{2 n-1}$ is degree $p^{r}$ on the bottom cell and we have the factorization

$$
\Omega^{2} p^{r}=\Sigma^{2} \cdot \pi_{r}: \Omega^{2} S^{2 n+1} \stackrel{\pi_{r}}{\rightarrow} S^{2 n-1} \stackrel{\Sigma^{2}}{\rightarrow} \Omega^{2} S^{2 n+1} .
$$

Remark 20.3 In fact, the above theorem is true for $p=3$ [17].

Since $\Omega^{2} p^{r}$ induces multiplication by $p^{r}$ on homotopy groups, setting $r=1$ in the above and recalling that $\pi_{*}\left(S^{1}\right)$ has no torsion yields

Corollary 20.4 If $p$ is an odd prime, then $p^{n}$ annihilates the p primary component of $\pi_{*}\left(S^{2 n+1}\right)$.

This result is best possible since Gray [7] has shown that $\pi_{*}\left(S^{2 n+1}\right)$ contains infinitely many elements of order $p^{n}$.

Remark 20.5 The case $n=1$ of the above corollary was first proved by Selick [22] by different methods which involved the $p$-th Hopf invariant.

\section{References}

1. Adams, J.F.: On the nonexistence of elements of Hopf invariant one. Ann. Math. 72, $20-104$ (1960)

2. Browder, W.: Torsion in H-spaces. Ann. Math. 74, 24-51 (1961)

3. Cartan, H., Eilenberg, S.: Homological Algebra. Princeton University Press, Princeton (1956)

4. Cohen, F.R., Moore, J.C., Neisendorfer, J.A.: The double suspension and exponents of the homotopy groups of spheres. Ann. Math. 110, 549-565 (1979) 
5. Cohen, F.R., Moore, J.C., Neisendorfer, J.A.: Torsion in homotopy groups. Ann. Math. 109, 121-168 (1979)

6. Eilenberg, S., Moore, J.C.: Homology and fibrations I, coalgebras, cotensor product and its derived functors. Comment. Math. Helv. 40, 199-236 (1966)

7. Gray, B.I.: On the sphere of origin of infinite families in the homotopy groups of spheres. Topology $\mathbf{8}$, 219-232 (1969)

8. Hopf, H.: Uber die abbildungen von spharen niedriger dimensionen. Fundam. Math. 25, 427-440 (1935)

9. Kan, D.M.: A combinatorial definition of homotopy groups. Ann. Math. 67, 282-312 (1958)

10. Liulevicius, A.: The factorization of cyclic reduced powers by secondary cohomology operations. Mem. A.M.S. 42. American Mathematical Society (1962)

11. MacLane, S.: Homology. Springer, Berlin (1963)

12. Massey, W.: Exact couples in algebraic topology I. Ann. Math. 56, 363-396 (1952)

13. Massey, W.: Exact couples in algebraic topology II. Ann. Math. 57, 248-286 (1953)

14. May, J.P.: Simplicial Objects in Algebraic Topology. Van Nostrand (1967)

15. Moore, J.C.: The double suspension and p-primary components of the homotopy groups of spheres. Boll. Soc. Mat. Mexicana 1, 28-37 (1956)

16. Neisendorfer, J.A.: Primary homotopy theory. Mem. A.M.S. 232. American Mathematical Society (1980)

17. Neisendorfer, J.A.: 3-Primary exponents. Math. Proc. Camb. Phil. Soc. 90, 63-83 (1981)

18. Neisendorfer, J.A.: The exponent of a Moore space. In: Browder, W. (ed.) Algebraic Topology and Algebraic K-Theory, pp. 35-71. Princeton University Press, Princeton (1987)

19. Neisendorfer, J.A.: Algebraic Methods in Unstable Homotopy Theory. Cambridge University Press, Cambridge (2009)

20. Neisendorfer, J.A.: Homotopy groups with coefficients. J. Fixed Point Theory Appl. 8, 247-338 (2010)

21. Quillen, D.: Rational homotopy theory. Ann. Math. 90, 295-295 (1969)

22. Selick, P.S.: Odd primary torsion in $\pi_{k}\left(S^{3}\right)$. Topology 17, 407-412 (1978)

23. Serre, J.-P.: Lie Algebras and Lie Groups. Benjamin (1965)

24. Shimada, N., Yamanoshita, T.: On triviality of the mod p Hopf invariant. Jpn. J. Math. 31, 1-25 (1961)

25. Steenrod, N.E., Epstein, D.B.A.: Cohomology Operations. Ann. Math. Studies, vol. 50. Princeton University Press (1962)

26. Toda, H.: On the double suspension $E^{2}$. J. Inst. Polytech. Osaka City Univ. Ser. A 7, 103-145 (1956)

27. Whitehead, G.W.: On mappings into group-like spaces. Comment. Math. Helv. 28, 320-328 (1954)

28. Whitehead, G.W.: Elements of Homotopy Theory. Springer, Berlin (1978) 